\title{
1 Accounting for spatiotemporal errors of gauges: A critical step to evaluate
}

3 Guoqiang Tang ${ }^{\mathrm{a}, \mathrm{b}}$, Ali Behrangi ${ }^{\mathrm{c}, \mathrm{d}, \mathrm{b}}$, Di Long ${ }^{\mathrm{a}}$, Changming Li $^{\mathrm{a}}$, Yang Hong ${ }^{\mathrm{a}, \mathrm{e}}$

4 a State Key Laboratory of Hydroscience and Engineering, Department of Hydraulic Engineering,

5 Tsinghua University. Beijing, China

$6 \quad{ }^{\mathrm{b}}$ Joint Institute for Regional Earth System Science and Engineering and Department of Atmospheric

7 and Oceanic Sciences, University of California, Los Angeles, California, USA

$8{ }^{\mathrm{c}}$ Department of Hydrology and Atmospheric sciences, University of Arizona, Tucson, Arizona, USA

$9{ }^{\mathrm{d}}$ Jet Propulsion Laboratory, California Institute of Technology, Pasadena, California, USA

$10{ }^{\mathrm{e}}$ Department of Civil Engineering and Environmental Science, University of Oklahoma. Norman,

11 Oklahoma, USA

12 Corresponding Author 1: Yang Hong, Room A207, State Key Laboratory of Hydroscience and

13 Engineering, Department of Hydraulic Engineering, Tsinghua University, Beijing 100084, China.

14 Corresponding Author 2: Di Long, Room 224, New Hydraulic Engineering Building, Department of

15 Hydraulic Engineering, Tsinghua University, Beijing 100084, China.

16 *Email: hongyang@tsinghua.edu.cn

17 **Email: dlong@tsinghua.edu.cn

18 Phone: 8618601113856

19 A revised submission to Journal of Hydrology on February 12, 2017 
20 Abstract: Rain gauge observations are commonly used to evaluate the quality of satellite

21 precipitation products. However, the inherent difference between point-scale gauge

22 measurements and areal satellite precipitation, i.e. a point of space in time accumulation

23 v.s. a snapshot of time in space aggregation, has an important effect on the accuracy and

24 precision of qualitative and quantitative evaluation results. This study aims to quantify

25 the uncertainty caused by various combinations of spatiotemporal scales $\left(0.1^{\circ}-0.8^{\circ}\right.$ and

26 1-24h) of gauge network designs in the densely gauged and relatively flat Ganjiang River

27 basin, South China, in order to evaluate the state-of-the-art satellite precipitation, the

28 Integrated Multi-satellite Retrievals for Global Precipitation Measurement (IMERG). For

29 comparison with the dense gauge network serving as "ground truth", 500 sparse gauge

30 networks are generated through random combinations of gauge numbers at each set of

31 spatiotemporal scales. Results show that all sparse gauge networks persistently

32 underestimate the performance of IMERG according to most metrics. However, the

33 probability of detection is overestimated because hit and miss events are more likely

34 fewer than the reference numbers derived from dense gauge networks. A nonlinear error

35 function of spatiotemporal scales and the number of gauges in each grid pixel is

36 developed to estimate the errors of using gauges to evaluate satellite precipitation.

37 Coefficients of determination of the fitting are above 0.9 for most metrics. The error

38 function can also be used to estimate the required minimum number of gauges in each 
39 grid pixel to meet a predefined error level. This study suggests that the actual quality of

40 satellite precipitation products could be better than conventionally evaluated or expected,

41 and hopefully enables non-subject-matter-expert researchers to have better understanding

42 of the explicit uncertainties when using point-scale gauge observations to evaluate areal

43 products.

44 Key terms: Precipitation; IMERG; rain gauge; gauge density; evaluation; error function 


\section{1. Introduction}

46 Precipitation is one of the most critical components in the global water and energy cycles.

47 However, accurate measurement of precipitation has been challenging due to its high

48 spatiotemporal variations. In general, there are three main approaches to observing

49 precipitation, i.e., rain gauges, weather radars, and satellite sensors (Li et al., 2013).

50 Novel approaches to precipitation estimation are also being developed and provide more

51 alternatives based on various data sources, such as soil moisture and water storage

52 changes (Behrangi et al., 2017; Ciabatta et al., 2015). Nevertheless, only satellite remote

53 sensing provides global coverage of precipitation estimation (Tian and Peters-Lidard, 54 2010).

55 Various multi-satellite precipitation products have been made available in recent years,

56 such as the Integrated Multi-satellitE Retrievals for Global Precipitation Measurement

57 (IMERG) (Huffman et al., 2017), Tropical Rainfall Measuring Mission (TRMM)

58 Multi-satellite Precipitation Analysis (TMPA) (Huffman et al., 2007), Climate Prediction

59 Center (CPC) MORPHing technique (CMORPH) (Joyce et al., 2004), and Global

60 Satellite Mapping of Precipitation (GSMaP) (Kubota et al., 2007). However, satellite

61 precipitation products are also subject to various systematic and random error sources,

62 including deficiencies in algorithms, inadequate spatiotemporal sampling, and instrument

63 limitations (Xie and Arkin, 1997). These errors are characterized by regional and seasonal

64 variations and could be propagated into studies that use the data (Hong et al., 2006). A 
65 large number of studies have been conducted to quantify errors of satellite precipitation

66 products for better applications and further improvements (Behrangi et al., 2011; Shen et

67 al., 2010; Tang et al., 2016b; Tang et al., 2016c; Yong et al., 2013).

68 Rain gauges are commonly employed to evaluate the performance of satellite

69 precipitation products because of the advantages of direct and accurate measurement,

70 sizeable spatial coverage, long time span, and simple data acquisition and processing.

71 However, rain gauges are also subject to errors such as the wind-induced undercatch,

72 trace amount, wetting and evaporation loss (Adam and Lettenmaier, 2003; Ma et al., 2015;

73 Yang and Ohata, 2001). In addition, accumulated precipitation of rain gauges is different

74 with satellite precipitation with temporal sampling errors resulting from repeated

75 temporal gaps, which contributes to uncertainties in the evaluation. Furthermore,

76 point-scale gauge observations can deviate far from true areal precipitation. For example,

77 Wood et al. (2000) indicated that the standard error of precipitation based on a single

78 gauge is around $33 \%$ in a $2 \times 2 \mathrm{~km}$ grid pixel. Jensen and Pedersen (2005) found that

79 variation in accumulated precipitation from 9 gauges in a $500 \times 500 \mathrm{~m}$ pixel is even up to

$80100 \%$ between neighboring gauges over a 4-day period. Villarini et al. (2008) suggested

81 that over 25 gauges are needed to make the error of average rainfall within $20 \%$ of the

82 actual rainfall in a pixel of $\sim 200 \mathrm{~km}^{2}$ at the 15 min scale. Therefore, the mismatch

83 between continuously accumulating point-scale gauge observations and snapshot areal

84 satellite precipitation has a marked impact on evaluation results. 
85 However, currently there seems to be no better alternatives compared to rain gauges for

86 the evaluation of satellite precipitation products. Unfortunately, publicly accessible rain

87 gauges are very sparse in most regions of the world, especially mountainous areas such as

88 the Tibetan Plateau (Gao and Liu, 2013). Interpolation methods such as inverse distance

89 weighting, nearest neighbor, and kriging can be employed to transfer point-scale

90 observations to areal estimates (Vicente-Serrano et al., 2003). However, interpolation also

91 brings additional uncertainties and the quality of interpolated precipitation in grid pixels

92 without gauges is typically lower than that in grid pixels with gauges (Tang et al., 2016b).

93 To ensure the reliability of results, assessment of satellite products is often conducted in

94 grid pixels containing at least one gauge or close to gauges (Asong et al., 2017; Cohen

95 Liechti et al., 2012; Salio et al., 2015; Shen et al., 2010; Tang et al., 2016b; Xu et al.,

96 2017). To acquire more representative areal precipitation using rain gauges, some studies

97 set a minimum number ( $>$ 1) of gauges contained in each grid pixel (Tan and Duan, 2017;

98 Xue et al., 2013; Yong et al., 2014; Yong et al., 2010; Yong et al., 2013). For instance,

99 Yong et al. (2014; 2010; 2013) only selected grid pixels containing at least two gauges to

100 evaluate the performance of TMPA 3B42 and 3B42RT products in two basins at $0.25^{\circ}$

101 and daily scales. Some studies are conducted in regions with highly dense rain gauges

102 and can achieve more reliable results (Tan et al., 2016; Tan and Duan, 2017). However,

103 such regions are universally small in area, and similar studies are typically not possible in 104 most regions of the world. 
105 Despite these efforts to involve as many gauges as possible, only a few studies

106 investigate the uncertainties of using rain gauges to evaluate satellite or radar

107 precipitation (Mandapaka et al., 2009; Rudolf et al., 1994). For radar rainfall evaluation,

108 some studies use highly dense gauge networks to evaluate errors and spatial correlation in

109 small experimental regions, and explore the effect of gauge configuration (Mandapaka et

110 al., 2010; Villarini et al., 2008). In addition to radar estimation errors, gauge sampling

111 errors also contribute to difference between radar and rain gauge rainfall, and generally

112 decrease with increasing time scales (Peleg et al., 2013). Similarly, for satellite

113 precipitation evaluation, it is important to investigate the reliability of evaluation results

114 based on rain gauges, and understand the accuracy of metrics used to indicate the quality

115 of evaluated objects.

116 Therefore, the objectives of this study are twofold: (1) comparing the metrics of satellite

117 precipitation products based on sparse and highly dense gauge networks at $0.1^{\circ}-0.8^{\circ}$ and

118 1-24 h scales from June to August 2014 in the Ganjiang River basin in South China; and

119 (2) proposing an error function of spatial scales, temporal scales, and gauge numbers to

120 characterize the errors of using rain gauges to evaluate satellite precipitation products. 


\section{2. Study area, data sets, and methods}

\section{$123 \quad 2.1$ Study area}

124 The study area covers $24^{\circ} \mathrm{N}-29.4^{\circ} \mathrm{N}$ and $113^{\circ} \mathrm{N}-117^{\circ} \mathrm{N}$, which is centered by the

125 Ganjiang River basin, China, with a highly dense rain gauge network (Figure 1). For

126 simplicity, the entire study area is referred to as the Ganjiang River basin. The

127 topography of this region is characterized by hills in the southern part and alluvial plains

128 in the northwestern part with elevations ranging from $7 \mathrm{~m}$ to $1997 \mathrm{~m}$. The climate is

129 dominated by the East Asian monsoon (Tang et al., 2016c). Previous studies indicated

130 that satellite precipitation products including TMPA and IMERG perform well in the

131 Ganjiang River basin from statistical and hydrological perspectives (Hu et al., 2014; Tang

132 et al., 2016c).

133

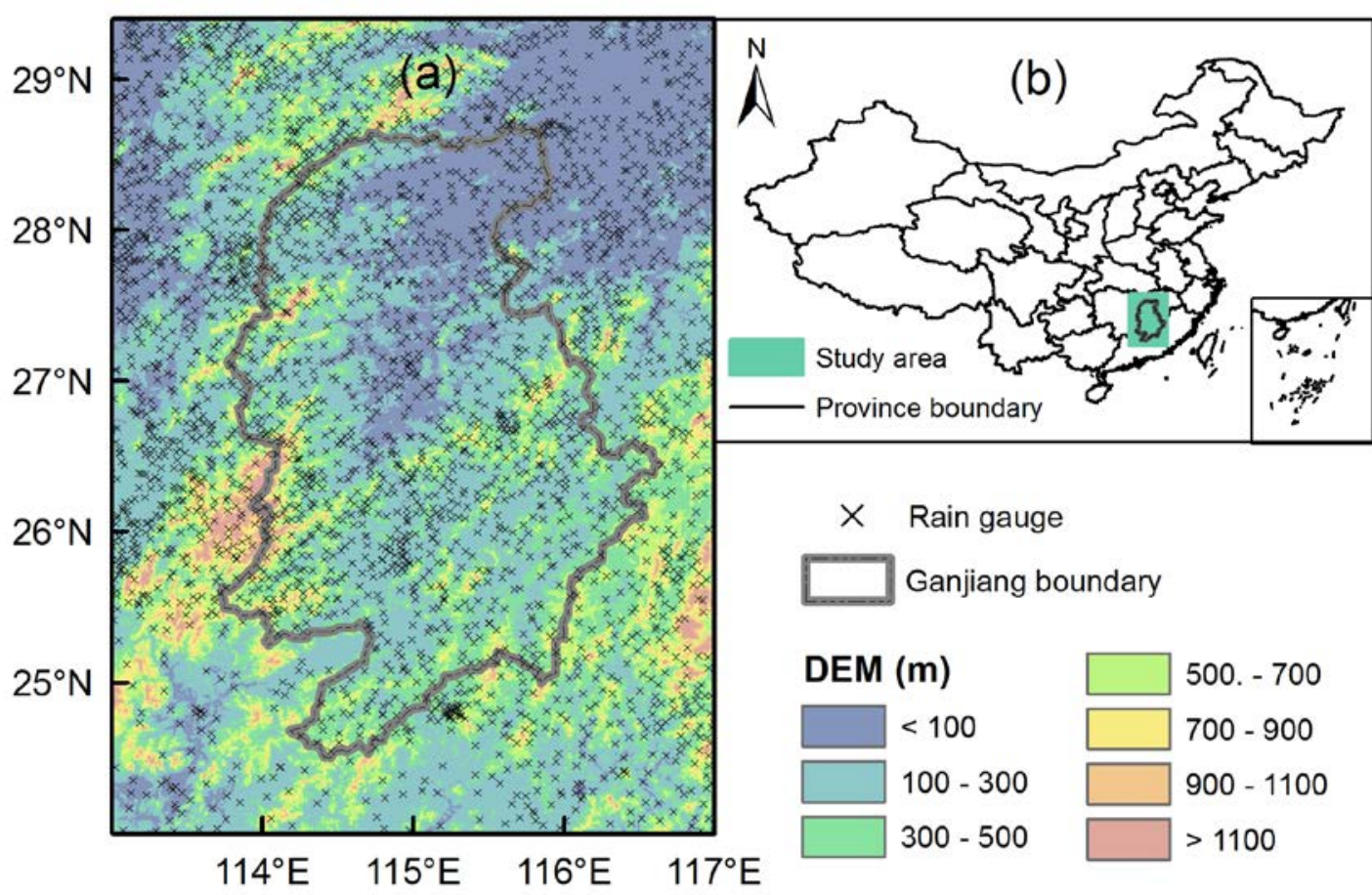


134 Figure 1. (a) Spatial distribution of rain gauges, and DEM of the study area which is centered by the

135 Ganjiang River basin. (b) Location of the study area in China.

\section{$136 \quad 2.2$ Data sets}

\section{$137 \quad$ 2.2.1 Rain gauges}

138 Hourly rain gauge data were acquired from the China Meteorological Administration

139 (CMA) (Shen et al., 2014). The total number of gauges is 3032, which is large for the

140 study area with a latitude span of $5.4^{\circ}$ and longitude span of $4^{\circ}$. The study period is from

141 June to August 2014, corresponding to the warm season in the Ganjiang River basin,

142 because rain gauges are less reliable in cold seasons. The hourly gauge observations have

143 undergone preliminary quality control such as extreme rainfall intensity check (Shen et

144 al., 2014). However, due to various unpredictable instrument problems, further quality

145 control is performed to ensure persuasive validation of satellite products (refer to Section $146 \quad 2.3 .1)$

\section{$147 \quad$ 2.2.2 IMERG products}

148 The Global Precipitation Measurement (GPM) mission aims at providing the next 149 generation of global precipitation products after the TRMM satellite was

150 decommissioned in 2015. IMERG provides combined-satellite Level 3 products at $0.1^{\circ}$

151 and 30 min resolutions. Compared with TMPA 3B42 and 3B42RT, by and large, IMERG

152 improves the accuracy of precipitation estimation (Ma et al., 2016; Tang et al., 2016a). 
153 IMERG has three products: the near-real-time Early and Late run, and the post-real-time

154 Final run. The Final run incorporates gauge data from the Global Precipitation

155 Climatology Centre (GPCC) and is characterized by high accuracy but large latency. We

156 used the IMERG Late run product (referred as IMERG-L for simplicity) to avoid the

157 problem of gauge dependence. GPCC and CMA have overlapping gauges although the

158 number is minimal compared to the CMA gauge number. However, at coarse spatial

159 scales (e.g., $0.8^{\circ}$ ), many grid pixels could contain a few GPCC gauges and affect the

160 evaluation results.

\section{$161 \quad 2.3$ Methods}

\section{$162 \quad$ 2.3.1 Rain gauge quality control}

163 Three quality control strategies were used, including missing data check, zero value

164 check, and correlation check. For missing data check, if the proportion of missing data in

165 the hourly data series exceeds $10 \%$, the rain gauge is excluded. To conduct zero value

166 check and correlation check, rain gauges within each $0.2^{\circ} \times 0.2^{\circ}$ grid pixel are grouped

167 into pairs. Note that the $0.2^{\circ}$ scale is only used for quality control. It is reasonably

168 assumed that the hourly precipitation observations of all gauges in the same pair should

169 not exhibit distinct climatological differences. For zero value check, we calculated ratios

170 of zero values to the number of all samples for all the gauges and the mean ratio for each

171 data pair. For each pair, a gauge is removed if its zero ratio is larger than 1.5 times or

172 smaller than 0.5 times that of the mean zero ratio. This problem arises probably due to (1) 
173 some missing values being recorded as zero, and (2) format issues in some data records.

174 For correlation check, we first calculated the correlation coefficient (CC) between each

175 gauge and all the other gauges belonging to the same pair. Then, the mean CC was

176 acquired for each gauge. A gauge is excluded if its mean CC is lower than the predefined

177 threshold (0.4) determined by trial and error. The correlation problem could be attributed

178 to less-obvious instrument anomalies.

179 As a result, 347 gauges which account for $\sim 11.5 \%$ of the total number, were excluded

180 after the three steps of quality control. We also validated the effectiveness of the quality

181 control strategies by manually checking the hourly data series of those excluded gauges.

182 Results indicate that most of these gauges were indeed subject to quality problems, and

183 could cause nonnegligible errors in the study.

\section{$184 \quad$ 2.3.2 Metrics}

185 To comprehensively assess the performance of satellite precipitation products, we utilized

186 six metrics. CC denotes the correlation between rain gauges and satellite products.

187 Normalized root mean square error (NRMSE) and relative bias (BIAS) denote the error

188 characteristics of satellite products. Instead of RMSE, NRMSE is adopted because it

189 avoids increasing values with increasing temporal scales. Their formulas are

$190 \mathrm{CC}=\frac{\sum_{i=1}^{n}\left(S_{i}-\bar{S}\right) \cdot\left(G_{i}-\bar{G}\right)}{\sqrt{\sum_{i=1}^{n}\left(S_{i}-\bar{G}\right)^{2} \cdot\left(G_{i}-\bar{G}\right)^{2}}}$ 
$191 \quad$ BIAS $=\frac{\sum_{i=1}^{n}\left(S_{i}-G_{i}\right)}{\sum_{i=1}^{n} G_{i}}$

192 NRMSE $=\frac{\sqrt{\frac{1}{n} \cdot \sum_{i=1}^{n}\left(S_{i}-G_{i}\right)^{2}}}{\bar{G}}$

193 where $n$ is the number of samples, $S_{i}$ is satellite estimated precipitation, $G_{i}$ is gauge

194 observed precipitation, and $\bar{S}$ and $\bar{G}$ are their mean values, respectively. The perfect

195 values are 1 for CC, and 0 for BIAS and NRMSE, respectively.

196 In addition, three contingency metrics were used, including probability of detection (POD)

197 representing the ratio of events correctly detected by satellite products, false alarm ratio

198 (FAR) representing the ratio of events falsely reported by satellite products, and critical

199 success index (CSI) as a function of POD and FAR providing more balanced judgement

200 of satellite products. To calculate the three contingency metrics, we used a threshold to

201 determine rain or no rain $(R N)$ which was defined as $0.1 \mathrm{~mm}$ for $1 \mathrm{~h}$. Thresholds for other

202 temporal scales (3-24 h) were accumulated from the hourly threshold. For example, the

203 threshold is $0.3 \mathrm{~mm}$ at the $3 \mathrm{~h}$ scale. Uniform thresholds for all temporal scales are also

204 tested in Section 3.3.3. Formulas of POD, FAR, and CSI are given as follows:

$205 \quad \mathrm{POD}=\frac{H}{H+M}$

$206 \quad \mathrm{FAR}=\frac{F}{H+F}$

$207 \quad \mathrm{CSI}=\frac{H}{H+M+F}=\frac{1}{1 /(1-F A R)+1 / P O D-1}$

208 where $H$ is the number of hits, $M$ is the number of misses, and $F$ is the number of false 
209 alarms. A hit occurs when both gauge and satellite precipitation is larger than $R N$. A miss

210 occurs when gauge precipitation is larger than $R N$ while satellite precipitation is smaller

211 than $R N$. A false alarm occurs when gauge precipitation is smaller than $R N$ while satellite

212 precipitation is larger than $R N$. The perfect values are 1 for POD and CSI, and 0 for FAR,

213 respectively.

\section{$214 \quad$ 2.3.3 Quantifying the uncertainties in evaluation}

\section{5 (1) Generating sparse gauge networks}

216 In this study, a sparse gauge network is defined as the one that the number of gauges in

217 each grid pixel is small such that it cannot well represent areal precipitation. For example,

218 if a $0.25^{\circ}$ grid pixel only contains one gauge, it is definitely sparse. A dense gauge

219 network is the one that can be taken as the closest to true precipitation. However, the

220 dense networks in this study could also contain uncertainties caused by insufficient

221 densities and instrument errors (e.g., undercatch). Like many previous studies (Tang et al.,

222 2016c; Yong et al., 2014; Yong et al., 2013), we did not correct undercatch by gauges

223 which has small effect in the study region.

224 Sparse gauge networks are generated from the dense gauge network in the Ganjiang

225 River basin. The spatial scales (s) are from $0.1^{\circ}$ to $0.8^{\circ}$, and the temporal scales $(t)$ are

226 from $1 \mathrm{~h}$ to $24 \mathrm{~h}$. Although the $s$ of most satellite precipitation products is not larger than

$2270.25^{\circ}$, the resolution of some reanalysis products such as ERA-Interim can be coarser.

228 For an arbitrary combination of $s$ and $t$, only grid pixels $\left(G_{s t}\right)$ containing more gauges 
229 than a predefined threshold $\left(T H_{s t}\right)$ are involved in the evaluation to represent the dense

230 network. $T H_{s t}$ should be as large as possible so that the average precipitation of all

231 gauges in each grid pixel can approximate true areal precipitation. $T H_{s t}$ is determined

232 through sensitive analysis in Section 3.2.

233 The spatial distribution of $G_{s t}$ in the study area is not necessarily even, which does not

234 affect the results because we focus only on the differences between sparse and dense

235 networks. A sparse gauge network $\left(G_{s t}^{\prime}\right)$ was generated by randomly selecting a certain

236 number $\left(N_{s t}\right)$ of gauges in each grid pixel of $G_{s t}$. Therefore, $G_{s t}^{\prime}$ has the same grid

237 pixels as $G_{s t}$, whereas the number of gauges in each grid pixel is $N_{s t}$ which is much

238 smaller than $T H_{s t}$. The locations of the $N_{s t}$ gauges at the sub-grid scale could also be

239 uneven. For example, if these gauges are all located at the corner of the pixel, the mean

240 precipitation of $N_{s t}$ gauges could deviate far from true areal precipitation. To reduce

241 such uncertainties, we randomly generated 500 independent sparse gauge networks

242 (referred to as $G_{s t}^{500}$ ). The number 500 was determined through sensitivity analysis. We

243 found that the results remain almost the same as the number of independent sparse gauge

244 networks changes from 100 to 1000 . Finally, 500 was selected for the balance between

245 sample numbers and computational efficiency.

246 (2) Estimating the error of metrics based on sparse gauge networks

247 For each combination of $s$ and $t$, satellite precipitation was resampled from the IMERG-L

248 product at the original $0.1^{\circ}$ and 30 min resolutions. Specifically, precipitation at coarser 
249 spatial scales is averaged from $0.1^{\circ}$ scale, and precipitation at various temporal scales

250 was accumulated from 30 min. Satellite precipitation was evaluated using $G_{s t}$ as

251 benchmark. We presume that the six metrics $\left(\mathrm{Met}_{\mathrm{T}}\right)$ were close to the true metric values

252 due to the large number of gauges in each grid pixel. Then, metrics based on sparse gauge

253 networks $\left(\mathrm{Met}_{s}\right)$ were derived through evaluation of satellite precipitation using $G_{s t}^{\prime}$. The

254 error of Met $_{S}$ is calculated as

$255 \Delta$ MET $=\frac{\text { Met }_{\mathrm{S}}-\mathrm{Met}_{\mathrm{T}}}{\mathrm{Met}_{\mathrm{T}}} \times 100 \%$

256 where Met represents CC, BIAS, NRMSE, POD, FAR and CSI. For example, $\mathrm{CC}_{\mathrm{T}}$ and

$257 \mathrm{CC}_{\mathrm{S}}$ represent the $\mathrm{CC}$ based on dense gauge networks $\left(G_{s t}\right)$ and sparse gauge networks

$258\left(G_{s t}^{\prime}\right)$, respectively. The error of $\mathrm{CC}_{\mathrm{S}}$ benchmarked by $\mathrm{CC}_{\mathrm{T}}$ is $\Delta \mathrm{CC}$. For $G_{s t}^{500}$

259 comprising $500 G_{s t}^{\prime}, \Delta \mathrm{MET}^{500}$ is acquired. $\triangle \mathrm{MET}$ is the mean of $\triangle \mathrm{MET}^{500}$, and

260 represents the bias of using sparse gauge networks to evaluate satellite products.

\section{1 (3) Error function}

262 Hong et al. (2006) proposed a power-law function of $s$, $t$, rain intensity, and sampling

263 frequency to express the error of precipitation estimates. This study aims at expressing

264 the error of metrics, which nonlinearly changes with $s, t$, and $N_{s t}$. Following the form of

265 Hong et al. (2006) which shares some similarities with this study, a nonlinear error

266 function of $s, t$ and $N$ is developed to estimate $\Delta$ MET.

$267 \quad \Delta \mathrm{MET}=a \cdot s^{b} \cdot t^{c} \cdot N_{s t}{ }^{d}$ 
268 where $a, b, c$, and $d$ are parameters. Different metrics exhibit different error patterns and

269 thus different signs of the parameters. Eq. (8) can also be used to determine the density of

270 gauges that delivers a predefined accuracy level. To control the errors of metrics within a

271 specific range ( $\triangle \mathrm{MET}^{\mathrm{s}}$, e.g., $\left.\pm 5 \%\right)$, the minimum number of gauges $\left(N_{s t}{ }^{s}\right)$ required in

272 each grid pixel can be calculated using Eq. (9). An example of application is given in

273 Section 3.4.

$274 \quad N_{s t}{ }^{s}=\left(\frac{\Delta \mathrm{MET}^{s}}{a \cdot s^{b} \cdot t^{c}}\right)^{1 / d}$

275 3. Results

$276 \quad 3.1$ Spatial distribution of precipitation

277 Figure 2 compares the IMERG-L mean precipitation map with gauge observations in the

278 Ganjiang River basin. There is a notable precipitation band in the central area and a

279 hotspot in the southwest region, which is captured by both gauges and IMERG-L.

280 However, IMERG-L overestimates precipitation in the northwest area. The mean

281 IMERG-L and gauge precipitation is $5.68 \mathrm{~mm} / \mathrm{d}$ and $6.51 \mathrm{~mm} / \mathrm{d}$, respectively, indicating

282 that overall IMERG-L underestimates precipitation by $-12.67 \%$. The CC between

283 IMERG-L and gauge precipitation is 0.46 at the $0.1^{\circ}$ and $1 \mathrm{~h}$ scale, which is much higher

284 than that in western and northern China (Tang et al., 2016b). 
(a) Gauge

(b) IMERG-L

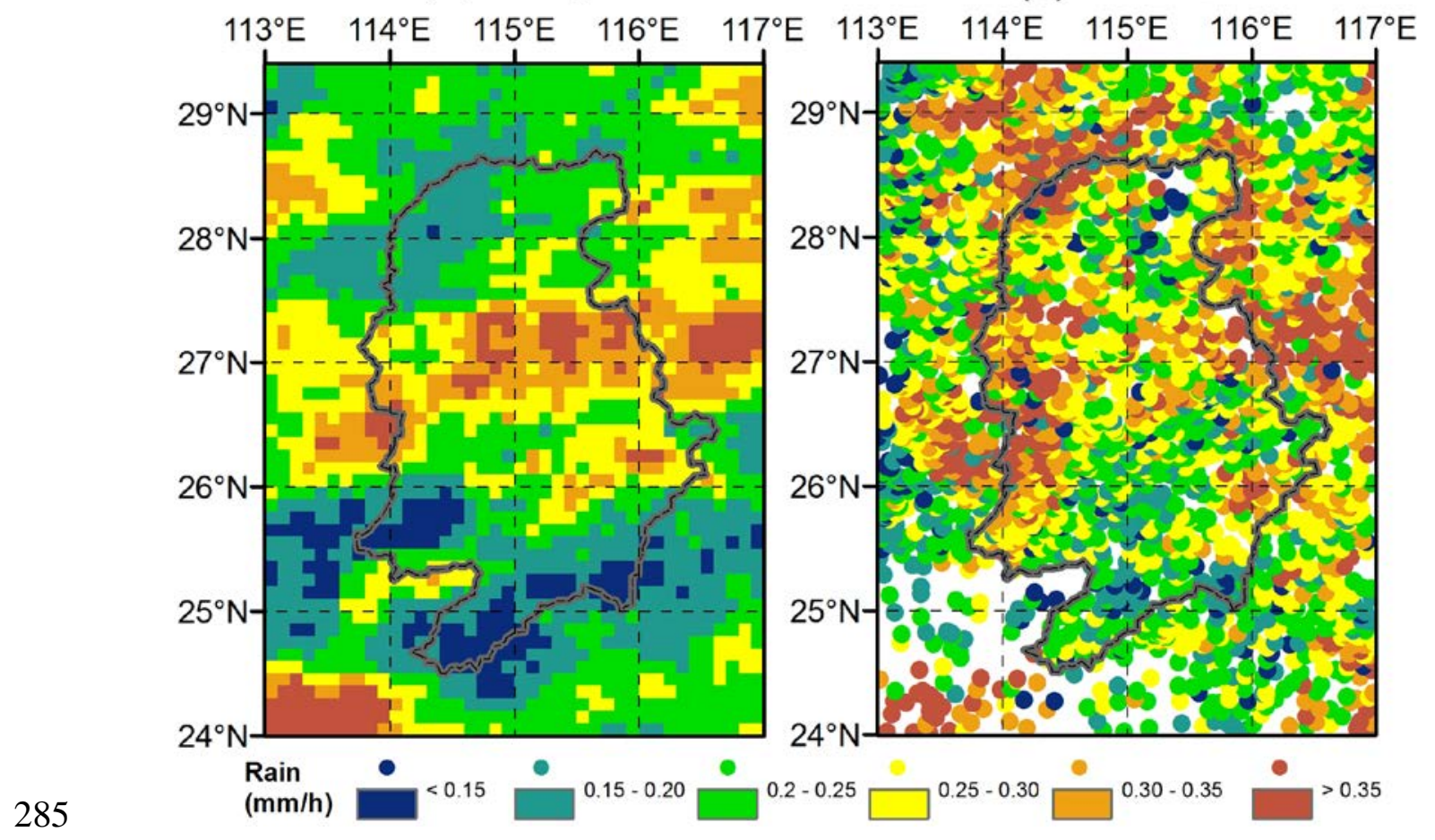

286 Figure 2. Precipitation distributions of (a) Gauge and (b) IMERG-L from June to August 2014 in the

287 Ganjiang River basin.

\section{3.2 Evaluating satellite products using highly dense gauges}

\section{3.2.1 Determining the number of gauges in each grid pixel}

290 A sensitivity analysis of $T H_{s t}$ was conducted to determine the minimum number of

291 gauges that can be taken as the closest to true areal precipitation in each grid pixel. The

292 spatial scales were from $0.1^{\circ}$ to $0.8^{\circ}$. The temporal scale was chosen to be $1 \mathrm{~h}$ because

$293 T H_{s t}$ at coarser temporal scales should be smaller than that at the hourly scale. As $T H_{s t}$

294 increases, the number of qualified grid pixels in the basin decreases. We first determine

295 the maximum $T H_{s t}$ at each spatial scale which is termed as Threshold 4 (T4). T4 is set 
296 as large as possible, but also keeps the number of qualified grid pixels within a 297 reasonable range. The process was completed by continued trial. Then, we obtained the 298 numbers of Thresholds 1-3 (T1-T3) by reducing the number of T4 gradually as shown in 299 Table 1. T1-T3 had smaller numbers of gauges in each grid pixel but larger number of 300 qualified pixels, which could be beneficial to acquiring comprehensive evaluation results.

301 Table 1 shows the numbers corresponding to T1-T4. $G_{s t}$ was acquired using T4 as $T H_{s t}$ 302 at all spatial scales. Then, $G_{s t}^{500}$ was acquired from $G_{s t}$ using T1, T2, and T3 as $N_{s t}$, 303 respectively, according to the method in Section 2.3.3. Finally, $\triangle \mathrm{MET}$ was obtained by 304 comparing $G_{s t}$ and $G_{s t}^{500}$, which represents the gap between T1-T3 and T4.

305 The smaller $\triangle \mathrm{MET}$, the closer the threshold to the "ideal" density, because marginal 306 benefits of increasing $T H_{s t}$ gradually decrease. Therefore, appropriate $T H_{s t}$ can be 307 determined according to the magnitude of $\triangle \mathrm{MET}$. If $\triangle \mathrm{MET}$ stabilizes, the 308 corresponding threshold can be regarded as dense enough. CC, NRMSE, and CSI were 309 selected to analyze $\triangle$ MET. Other metrics also have similar error characteristics although 310 they are not included in this section. The advantage of this method is that scenarios close

311 to actual evaluation studies are created, and both temporal and spatial variations of the

312 data series are considered. The method is better than just comparing mean areal

313 precipitation of gauges which cannot reflect the temporal variations of data series.

314 Table 1 Four sets of thresholds used to determine the minimum number of gauges that can 315 approximate true areal precipitation in each grid pixel at $0.1^{\circ}-0.8^{\circ}$ and $1 \mathrm{~h}$ scales. 


\begin{tabular}{cccccccccc}
\hline Spatial Scale & $\mathbf{0 . 1}^{\circ}$ & $\mathbf{0 . 2}^{\circ}$ & $\mathbf{0 . 3}^{\circ}$ & $\mathbf{0 . 4}^{\circ}$ & $\mathbf{0 . 5}^{\circ}$ & $\mathbf{0 . 6}^{\circ}$ & $\mathbf{0 . 7}^{\circ}$ & $\mathbf{0 . 8}^{\circ}$ \\
\hline Threshold 1 (T1) & 4 & 8 & 18 & 27 & 35 & 45 & 62 & 90 \\
Threshold 2 (T2) & 5 & 10 & 20 & 30 & 40 & 50 & 70 & 100 \\
Threshold 3 (T3) & 6 & 12 & 22 & 33 & 45 & 55 & 78 & 110 \\
Threshold 4 (T4) & 7 & 14 & 24 & 36 & 50 & 60 & 86 & 120 \\
\hline
\end{tabular}

316 Figure 3 shows that metrics based on T1-T3 are all slightly worse than those based on T4,

317 indicating that IMERG-L agrees better with denser gauge networks compared with sparse

318 gauge networks. $\triangle \mathrm{MET}$. decreases as the number increases from T1 to T3. Overall, the

319 errors of all the three metrics are very small, particularly at $0.3^{\circ}-0.8^{\circ}$ scales with the

320 absolute values of $\triangle \mathrm{MET}$. generally smaller than $1 \%$. At $0.1^{\circ}-0.2^{\circ}$ scales, $\triangle \mathrm{MET}$.

321 values are slightly larger, especially for T1, indicating that the numbers of gauges are

322 relatively insufficient. Although T3 and T4 are the closest to the ideal situation, we chose

323 T2 (see Table 1) as the $T H_{s t}$ because of its small $\triangle \mathrm{MET}$. within $\pm 1.5 \%$ benchmarked

324 by T4. More importantly, the number of qualified grid pixels based on T3 or T4 is too

325 small compared with that based on T2, which is undesirable when assessing satellite 326 precipitation.

327 We also used the variance reduction factor (VRF) to assess the accuracy of pixel-average 328 precipitation provided by T2 (Peleg et al., 2013; Villarini et al., 2008). Small VRF

329 indicates that the uncertainties of using pixel-average precipitation to represent true areal

330 precipitation are small. VRF values are generally smaller than $2 \%$ at $0.3^{\circ}-0.8^{\circ}$ and $3-24$ 
331 h scales (supplemental file: Figure S1). However, VRF is much larger at the $0.1^{\circ}$ scale

332 with values ranging between $5 \%$ and $7.6 \%$, indicating that the gauge network is still not

333 dense enough. This problem is inevitable because it is impractical to deploy a sufficient

334 dense network at the $0.1^{\circ}$ scale in a region as large as the Ganjiang River basin. Therefore,

335 the estimated metrics based on the dense gauge network can only approach but never

336 reach true metrics. $\triangle \mathrm{MET}$ calculated in this study is actually slightly smaller than the

337 actual errors, particularly at small spatial scales.

(a) Threshold

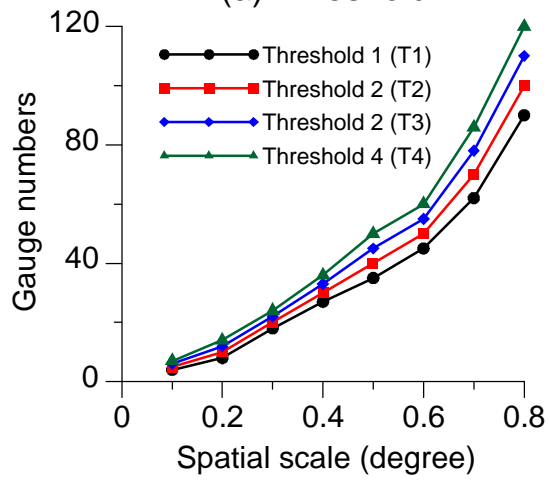

(c) NRMSE

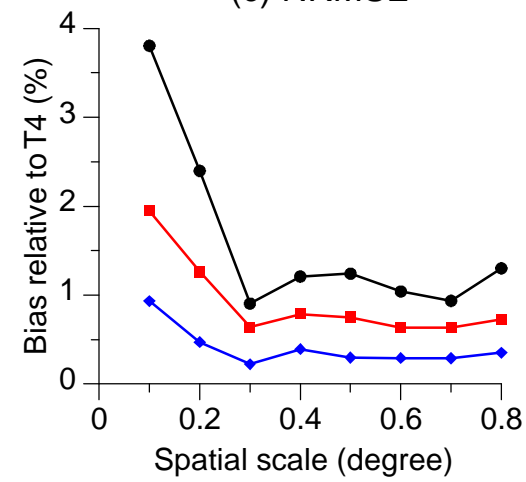

(b) $\mathrm{CC}$

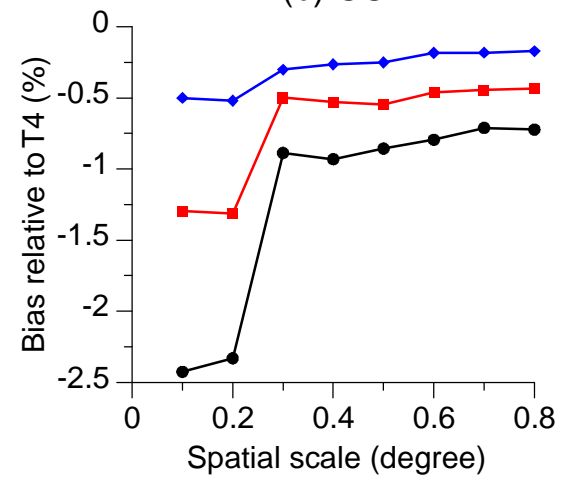

(d) CSI

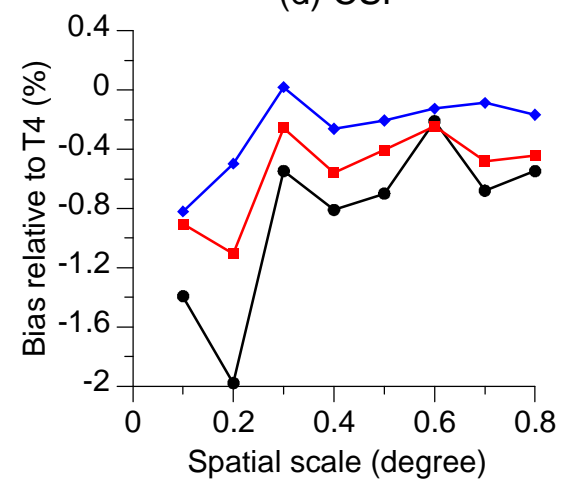

Figure 3. (a) Number of gauges corresponding to the four sets of thresholds (T1-T4) at $0.1^{\circ}-0.8^{\circ}$ 


\subsubsection{Metrics based on dense gauge networks}

343 Locations of $G_{s t}$ based on $\mathrm{T} 2$ at $0.1^{\circ}-0.8^{\circ}$ scales are shown in Figure 4. The spatial

344 distributions are uneven, with more pixels in the western and central parts and fewer

345 pixels in the eastern and southern parts. Therefore, the metrics derived in this study may

346 not represent the average situation in the Ganjiang River basin. Nevertheless, we focus on

347 the difference between sparse and dense gauge networks, which is not affected by this 348 problem.

(a) $0.1^{\circ}$

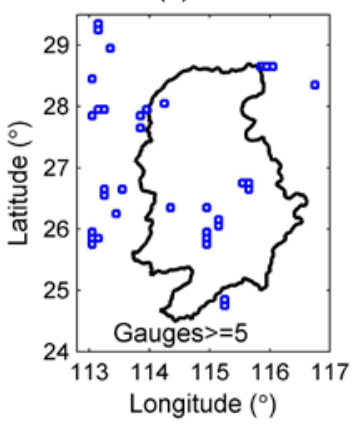

(e) $0.5^{\circ}$

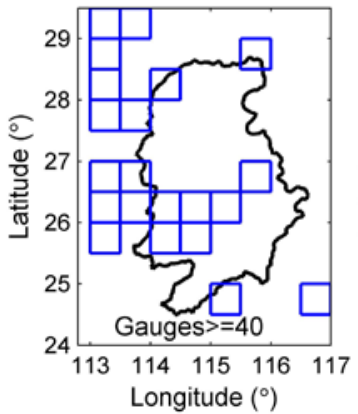

(b) $0.2^{\circ}$

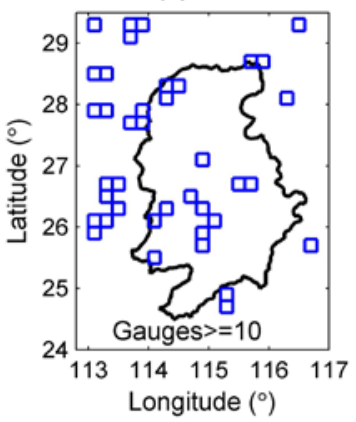

(f) $0.6^{\circ}$

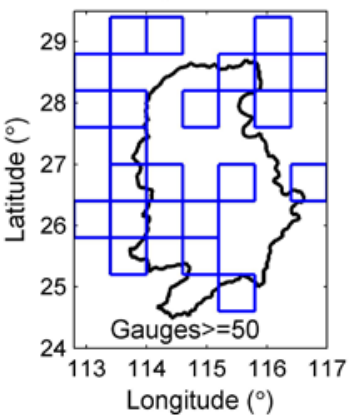

(c) $0.3^{\circ}$

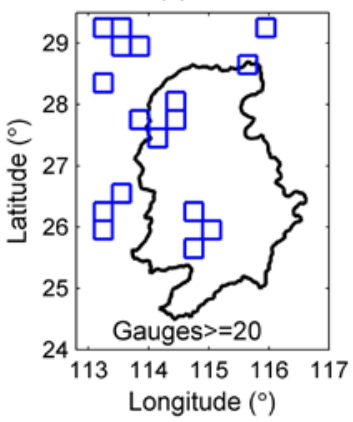

(g) $0.7^{\circ}$

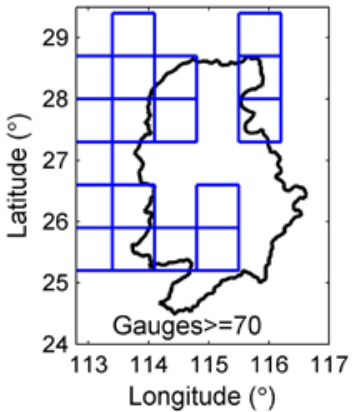

(d) $0.4^{\circ}$

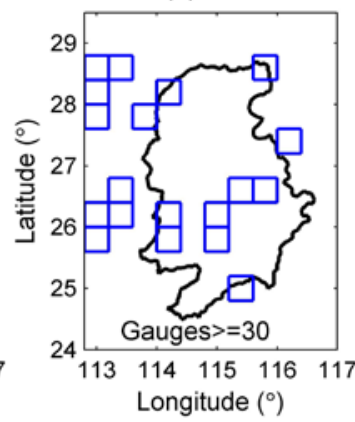

(h) $0.8^{\circ}$

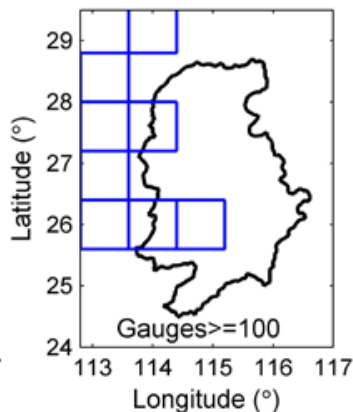

Figure 4. Locations of grid pixels (blue boxes) at $0.1^{\circ}-0.8^{\circ}$ scales in the study area. Only grid pixels

351 that contain a specific number of gauges as shown in the bottom of each subfigure are included.

352 As the spatial scale increases from $0.1^{\circ}$ to $0.8^{\circ}$ and the temporal scale increases from $1 \mathrm{~h}$

353 to $24 \mathrm{~h}$, most metrics are notably improved, i.e., increasing CC, POD, and CSI, and 
354 decreasing FAR and NRMSE (Figure 5). This result is consistent with previous studies on

355 the scale effect of the performance of satellite precipitation products (Hong et al., 2006;

356 Tang et al., 2016b). Note that we used NRMSE in this study (Figure 5b). RMSE will

357 exhibit a different trend with better performance (smaller values) as the spatial scale

358 decreases and the temporal scale increases because accumulated precipitation is used to

359 calculate the metric. Despite the difference, RMSE and NRMSE lead to similar errors of

360 metrics.

361 Different from the other metrics with clear and regular changing patterns, BIAS remains

362 almost the same when the temporal scale changes, and is irregular at different spatial

363 scales (Figure 5c) chiefly due to the shifting positions of the grid pixels that qualify at

364 each spatial scale (Figure 4). BIAS has strong spatial variation resulting in different

365 values at the eight spatial scales. Further comparison shows that grid pixels at different

366 scales exhibit similar BIAS values if these pixels are close to each other in location,

367 indicating that BIAS will remain consistent if calculated in the same regions. It should

368 also be noted that although the color contrast is conspicuous in Figure 5c, the extent of

369 BIAS is narrow ranging between $-8 \%$ and $-12 \%$. 
(a) $\mathrm{CC}$

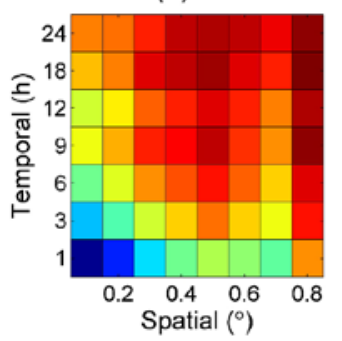

(d) POD

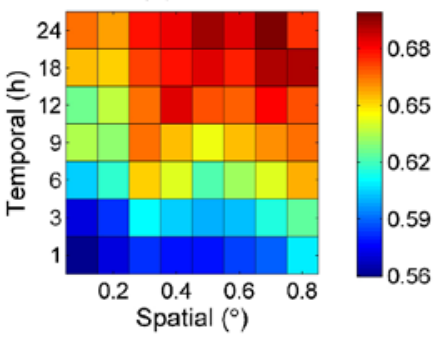

(b) NRMSE

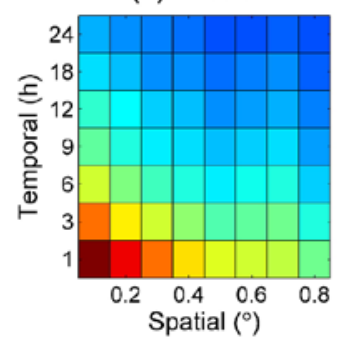

(e) FAR

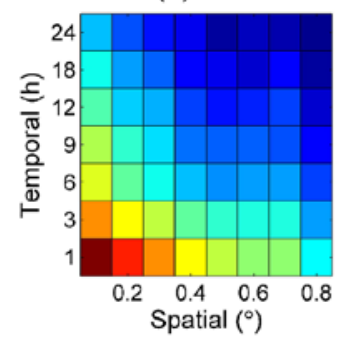

(c) BIAS
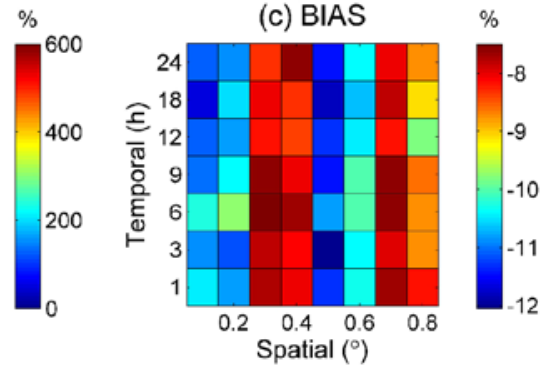

(f) $\mathrm{CSI}$
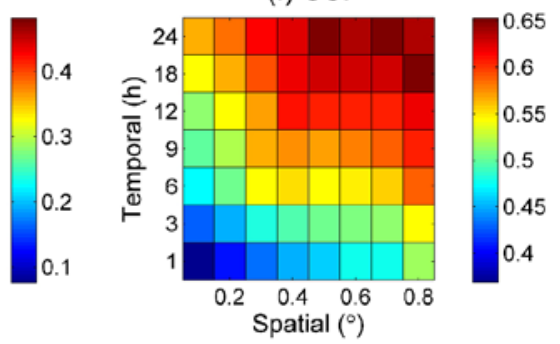

371 Figure 5. (a) CC, (b) NRMSE, (c) BIAS, (d) POD, (e) FAR, and (f) CSI based on highly dense gauge

372 networks at $0.1^{\circ}-0.8^{\circ}$ and $1-24$ h scales from June to August 2014 in the Ganjiang River basin. The

373 threshold used for rain/no rain determination is $0.1-2.4 \mathrm{~mm}$ at $1-24 \mathrm{~h}$ scales, respectively.

\section{$374 \quad 3.3$ Errors of metrics based on sparse gauge networks}

\section{$375 \quad$ 3.3.1 Histograms of metrics}

376 Figure 6 compares CCs based on 500 sparse gauge networks ( $G_{s t}^{500}$, refer to Section 2.3.3)

377 and $\mathrm{CC}_{\mathrm{T}}$ based on highly dense gauge networks $\left(G_{s t}\right)$. CCs was calculated in the same

378 grid pixels with $\mathrm{CC}_{\mathrm{T}}$ (Figure 4), while each grid pixel only contains one gauge. This

379 density of gauges is common in the evaluation of satellite precipitation products at $0.1^{\circ}$ or

$3800.25^{\circ}$ scales, although in reality grid pixels with larger sizes such as $0.8^{\circ}$ generally

381 contain more gauges.

$382 \mathrm{CC}_{\mathrm{T}}$ is much higher than $\mathrm{CC}_{\mathrm{s}}$ at all the temporal and spatial scales. As the temporal scale 
383 decreases and the spatial scale increases, the gap between $\mathrm{CC}_{\mathrm{T}}$ and CCs becomes larger.

384 Even at the $0.1^{\circ}$ and $1 \mathrm{~h}$ scales for which the gap is smallest, $\mathrm{CC}_{\mathrm{T}}$ is 0.46 while the mean

385 of CCs is only 0.39 with a negative $\Delta$ CC up to $-15 \%$. Moreover, each of the 500 CCs is

386 smaller than СС $\mathrm{s}$ at all spatiotemporal scales, indicating that the performance of satellite

387 products is always underestimated by a sparse gauge network without exception.

388 Increasing gauge numbers contained in each grid pixel can narrow but not eliminate the

389 gap. NRMSE, POD, FAR and CSI all exhibit similar phenomena (not shown).

390
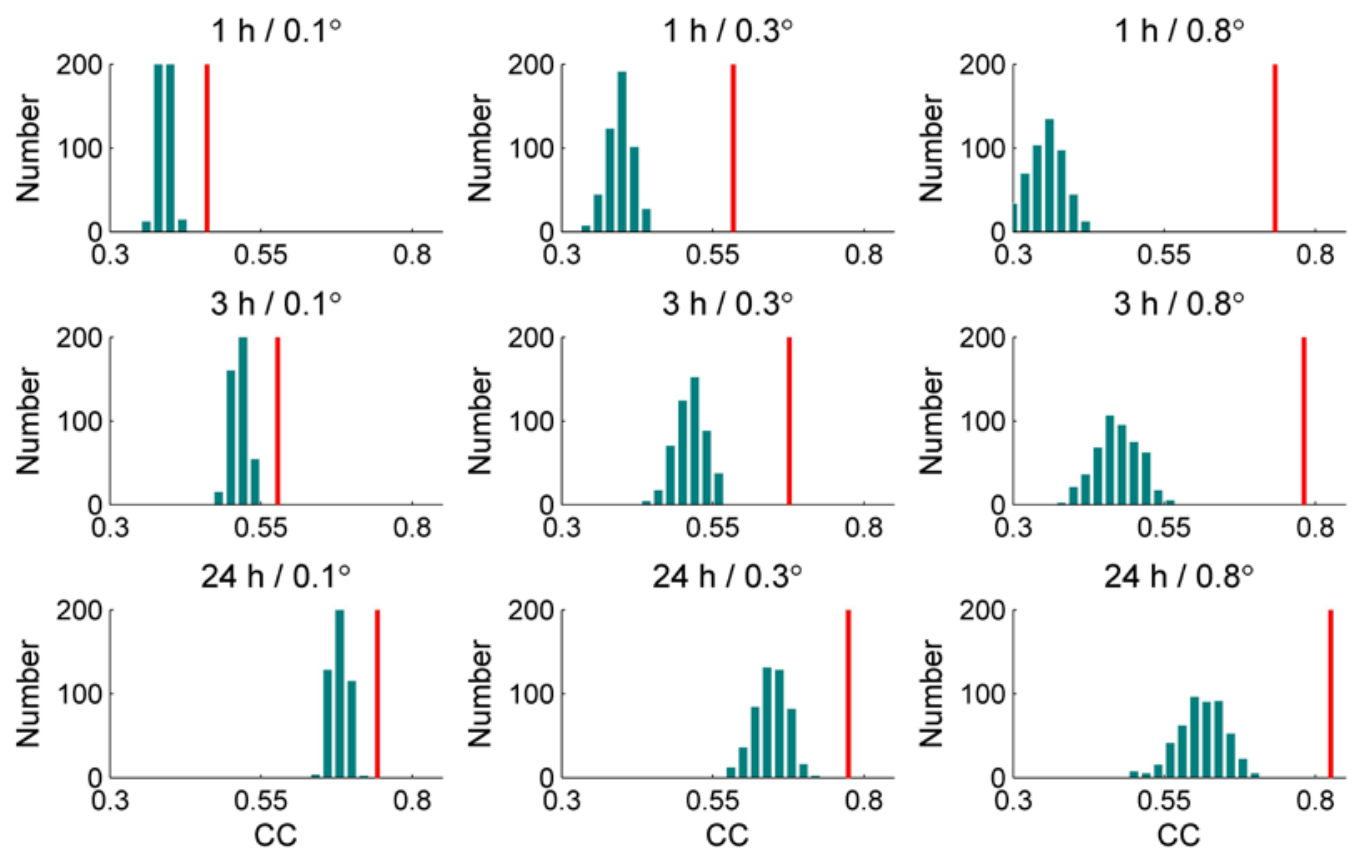

391 Figure 6. Histograms of CC between satellite precipitation estimates and rain gauge observations

392 based on 500 sparse gauge networks at $0.1^{\circ} / 0.3^{\circ} / 0.8^{\circ}$ and $1 / 3 / 24 \mathrm{~h}$ scales. Each grid pixel contains one

393 gauge. Red lines represent CC based on highly dense gauge networks.

394 BIAS is different from the other metrics as shown in Figure 7. BIAS $\mathrm{T}$ is located within 395 the extent of BIASs. However, the mean of BIASs is closer to zero compared to BIAST 
396 at all spatial scales. For example, at the $0.1^{\circ}$ and $1 \mathrm{~h}$ scale, BIAS $\mathrm{T}$ is $-10.45 \%$ while the

397 mean of BIASs is $-6.22 \%$. Furthermore, the BIASs of 198 networks among all the 500

398 sparse networks is positive, which means that evaluation based on $\sim 40 \%$ of the sparse

399 gauge networks will draw an opposite conclusion with the dense gauge network

400 concerning BIAS. Therefore, one should be cautioned with the nominal BIAS of satellite

401 products if the gauge network is relatively sparse. It is not certain whether satellite

402 products overestimate or underestimate precipitation if the BIAS value is close to zero.
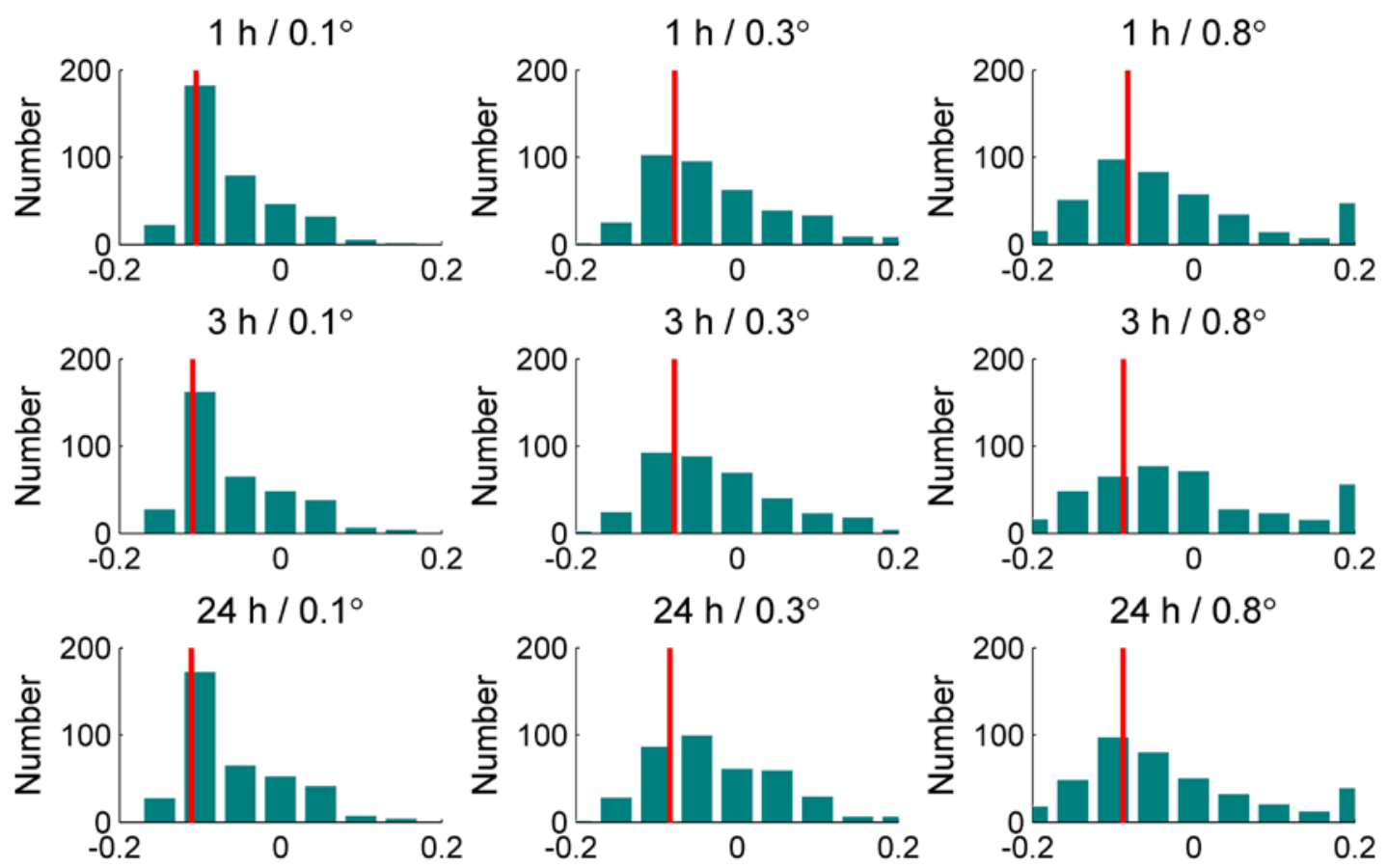

403

Bias

Bias

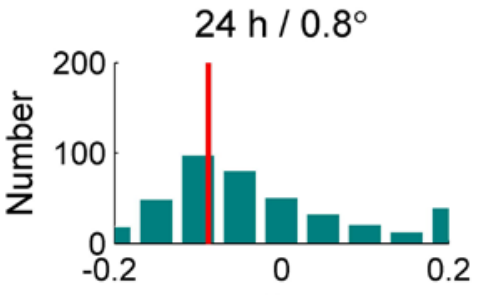

Bias

404 Figure 7. Same as Figure 6 but for BIAS.

\subsubsection{Errors of metrics at different spatiotemporal scales}

$406 \Delta$ MET of all the six metrics was calculated according to Eq. (7) at $0.1^{\circ}-0.8^{\circ}$ and $1-24 \mathrm{~h}$

407 scales as shown in Figure 8. Each grid pixel contains only one gauge. For CC, CSI and 
408 NRMSE, $\triangle$ MET becomes larger as the spatial scale increases and temporal scale

409 decreases (Figure 8a, b, and f). Overall, $\Delta \mathrm{CC}$ and $\Delta \mathrm{CSI}$. are moderate ranging between

$410-6 \%$ and $-50 \%$. $\triangle$ NRMSE is much larger, indicating that NRMSE is more prone to the

411 effect of gauge densities. For example, the overestimation of NRMSE ranges between

$41213.43 \%$ at the $0.1^{\circ}$ and $24 \mathrm{~h}$ scale and $134 \%$ at the $0.8^{\circ}$ and $1 \mathrm{~h}$ scale. $\Delta$ BIAS keeps

413 almost consistent for different temporal scales as expected, and is almost irregular as the

414 spatial scale increases from $0.1^{\circ}$ to $0.8^{\circ}$ (Figure 7c), which could be attributed to the

415 inconsistent locations of grid pixels at different spatial scales.

416 POD is insensitive to the number of gauges in each grid pixel. $\triangle \mathrm{POD}$ is very small 417 ranging between $-5 \%$ and $11 \%$. The pattern of $\Delta \mathrm{POD}$ is elusive (Figure $8 \mathrm{~d}$ ). At $0.1^{\circ}-0.7^{\circ}$

418 and 3-24 h scales, the pattern of $\triangle \mathrm{POD}$ is similar with that of $\triangle \mathrm{CC}, \triangle \mathrm{NRMSE}$, and $419 \Delta$ CSI. However, the values of $\triangle \mathrm{POD}$ are positive, indicating that POD based on sparse 420 networks is better than that based on dense networks. This is because a single gauge only 421 captures point-scale precipitation events which are far less than sufficient to represent 422 true areal events. Hit and miss events are fewer than the situation benchmarked by the 423 sparse gauge networks, leading to smaller $H$ and $M$ in Eq. (4). At 3-24 h scales, the effect 424 of the underestimation of $M$ is larger, resulting in the overestimation of POD. In contrast, 425 the effect of the underestimation of $H$ dominates at $1 \mathrm{~h}$ and $0.1^{\circ} / 0.2^{\circ}$ scales and POD is 426 slightly underestimated with negative $\triangle \mathrm{POD}$. At the $0.8^{\circ}$ scale, the pattern of $\triangle \mathrm{POD}$ is 427 not clear, probably because one gauge is too few for such a large scale and the calculated 
$H$ and $M$ contain large uncertainties.

429 In contrast to POD, FAR is the most sensitive metric, and is always overestimated by 430 sparse gauge networks with $\triangle \mathrm{FAR}$ ranging between $9 \%$ and $410 \%$. For example, at the $4310.1^{\circ}$ and $24 \mathrm{~h}$ scale which is common in the evaluation of IMERG-L products, FARs is 4320.28 while FAR $_{T}$ is only 0.20 with a $\triangle \mathrm{FAR}$ up to $40 \% . \triangle \mathrm{FAR}$ gets larger as the 433 temporal scale increases, which is contrary to $\triangle \mathrm{CC}, \triangle \mathrm{NRMSE}$, and $\triangle \mathrm{CSI}$. FAR is 434 positively correlated with $F$ and negatively correlated with $H$. Because a single gauge 435 tends to capture fewer precipitation events in each grid pixel, false alarms are much larger 436 than the actual situation leading to a much larger $F$. The large $F$ and small $H$ jointly 437 contribute to the large FAR and $\triangle F A R$ based on sparse gauge networks. As the temporal 438 scale increases, the ratio between $H$ and $F$ becomes smaller, leading to larger $\Delta \mathrm{FAR}$.

(a) $\mathrm{CC}$

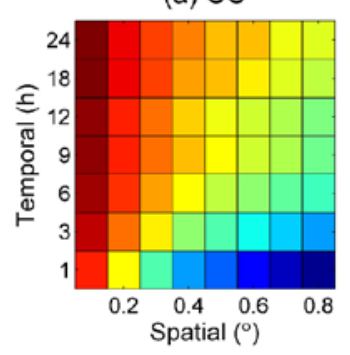

(d) POD

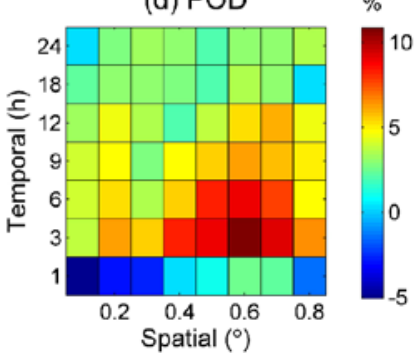

(b) NRMSE

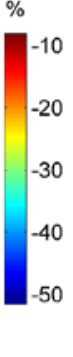

$\%$

(e) FAR
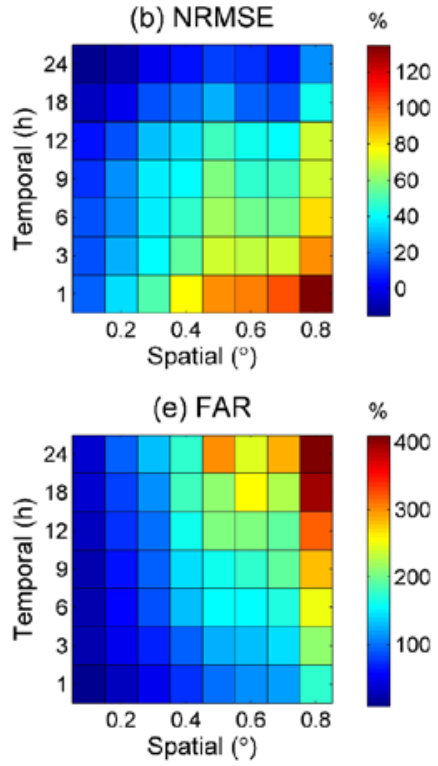

(c) BIAS

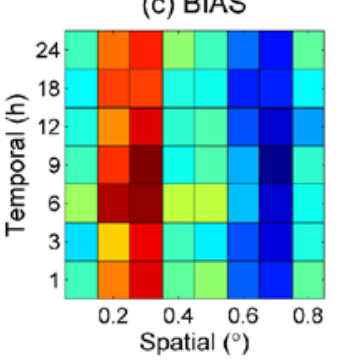

(f) $\mathrm{CSI}$
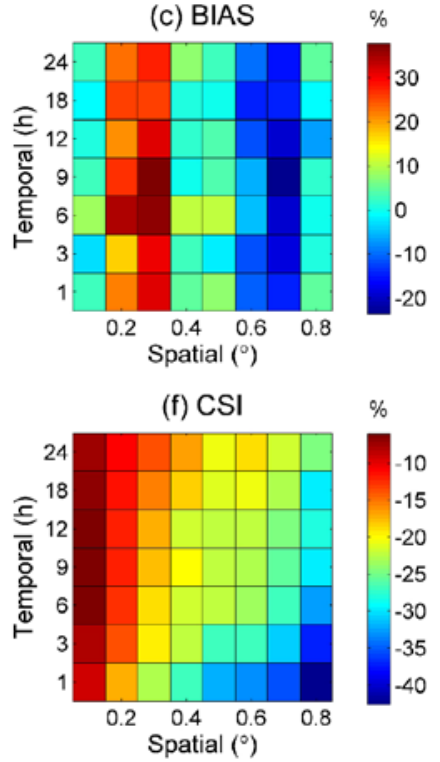

440 Figure 8. Errors of the six metrics based on sparse gauge networks at $0.1^{\circ}-0.8^{\circ}$ and $1-24 \mathrm{~h}$ scales 
441 relative to the dense gauge networks. Each grid pixel contains one gauge. Metrics based on highly

442 dense networks are utilized as a benchmark. The threshold used for rain/no rain determination is 0.1-

$443 \quad 2.4 \mathrm{~mm}$ at $1-24 \mathrm{~h}$ scales, respectively.

\section{3.3.3 Effect of rain/no rain thresholds and gauge numbers}

$445 R N$ values used to determine rain or no rain have a marked effect on contingency metrics,

446 including POD, FAR, and CSI. Figure 9 shows $\triangle \mathrm{POD}, \triangle \mathrm{FAR}$, and $\triangle \mathrm{CSI}$ at various

447 spatiotemporal scales using different $R N$ values from $0.05 \mathrm{~mm} / \mathrm{h}$ to $0.3 \mathrm{~mm} / \mathrm{h}$. Thresholds

448 at 3-24 h scales are accumulated from the hourly threshold. The patterns of $\triangle \mathrm{POD}$ show

449 notable variation, indicating that POD is more sensitive to $R N$ compared with FAR and

450 CSI. The patterns of $\triangle \mathrm{FAR}$ remain consistent at different $R N$ values, except for the

451 largest $R N(0.3 \mathrm{~mm} / \mathrm{h})$ for which the peak of $\Delta$ FAR shifts slightly. $\Delta$ CSI exhibits stable

452 patterns due to the advantage of combining POD and FAR.

453 In addition, we also checked the errors of metrics under the situation that all the temporal

454 scales adopted the same $R N$ as shown in Figure 10 (i.e., 0.1 and $0.2 \mathrm{~mm}$ for $1-24 \mathrm{~h}$ ).

455 Results indicate that the patterns of $\triangle \mathrm{FAR}$ and $\Delta \mathrm{CSI}$ change little, while the pattern of

$456 \triangle \mathrm{POD}$ shows more variation. Overall, FAR and CSI are less sensitive to the $R N$ threshold 457 values than POD. 

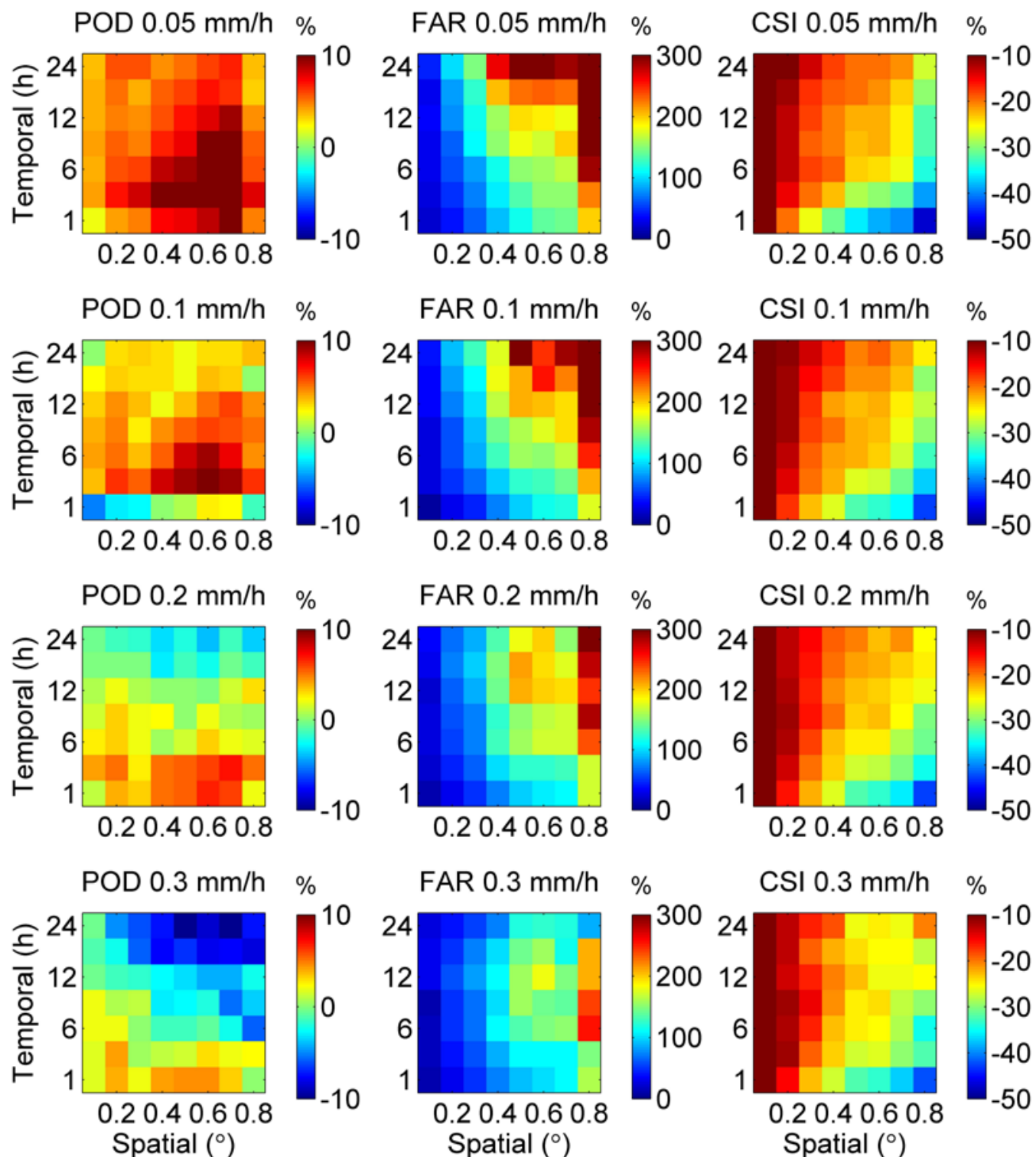

458
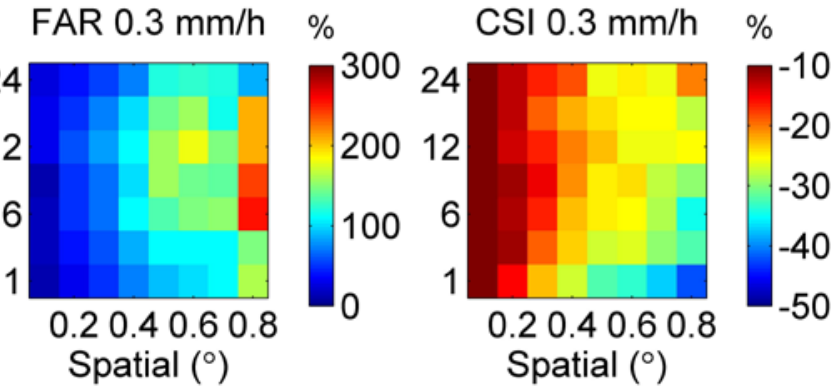

459 Figure 9. Same as Figure 8, but only for the three contingency metrics including POD, FAR and CSI.

460 The numbers behind metric names are thresholds $(0.05,0.1,0.2,0.3 \mathrm{~mm} / \mathrm{h})$ used to determine rain or

461 no rain. The thresholds for other time scales (3-24 h) are accumulated from the thresholds for $1 \mathrm{~h}$. 

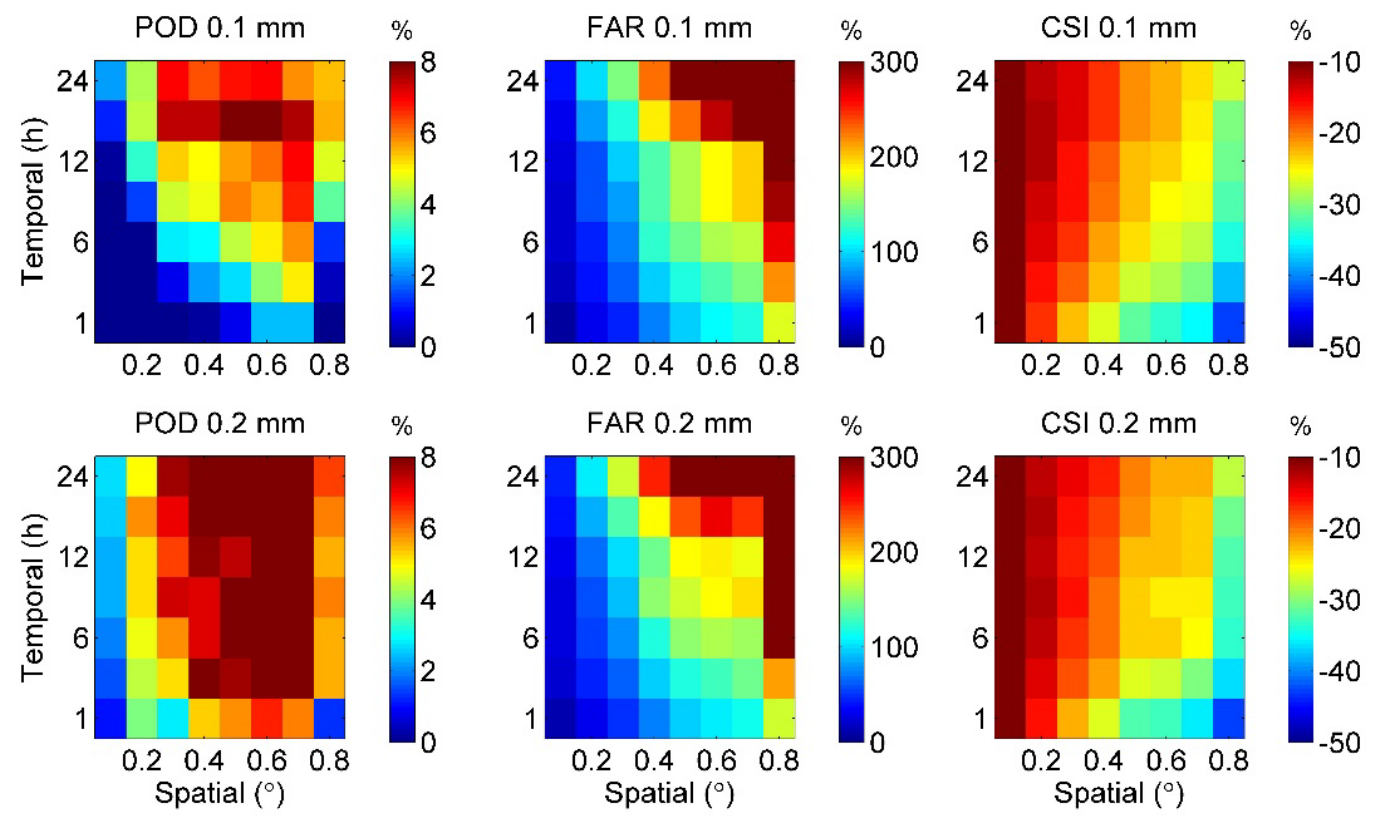

463 Figure 10. Same as Figure 9, but the thresholds are fixed at 1-24 h scales, i.e., $0.1 \mathrm{~mm}$ for the upper

464 row and $0.2 \mathrm{~mm}$ for the lower row.

465 In addition to $R N$, the effect of the number of gauges $\left(N_{s t}\right)$ contained in each grid pixel

466 was also investigated (Figure 11). $N_{s t}$ increases from one to $80 \%$ of $T H_{s t}$ (T2 in Table

467 1) to generate sparse gauge networks with different densities. BIAS is not included

468 because of its irregularities (Section 3.3.1 and 3.3.2). The combinations of $0.1^{\circ} / 0.8^{\circ}$ and

$4691 / 24 \mathrm{~h}$ scales were selected as representative examples. It is notable that $\triangle \mathrm{MET}$ of all

470 metrics decreases and tends to be zero as $N_{s t}$ increases. The curves of CC and CSI

471 almost overlap at large temporal or spatial scales (Figure 11b-d), indicating that the two

472 metrics have a similar sensitivity to gauge densities. $\triangle$ NRMSE and $\triangle F A R$ are positive

473 and increase rapidly as the temporal or spatial scales increase. POD always exhibits the

474 smallest difference. On the whole, the curves are smooth, which proves that it is feasible 
(a) $0.1^{\circ} 1 \mathrm{~h}$

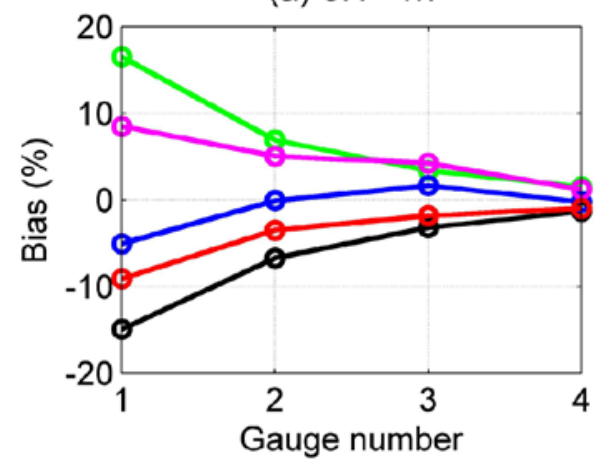

(c) $0.8^{\circ} 1 \mathrm{~h}$

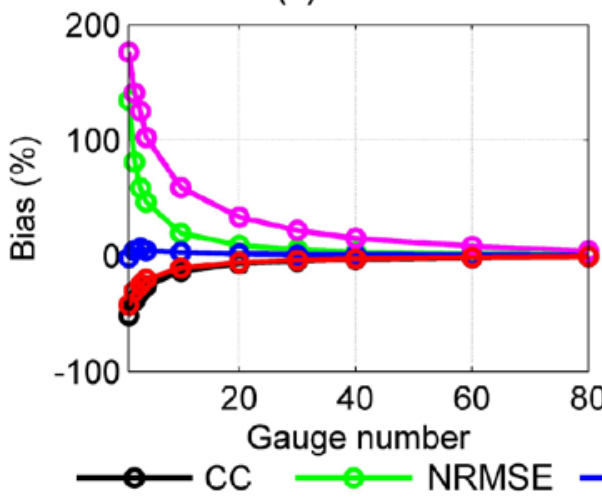

(b) $0.1^{\circ} 24 \mathrm{~h}$

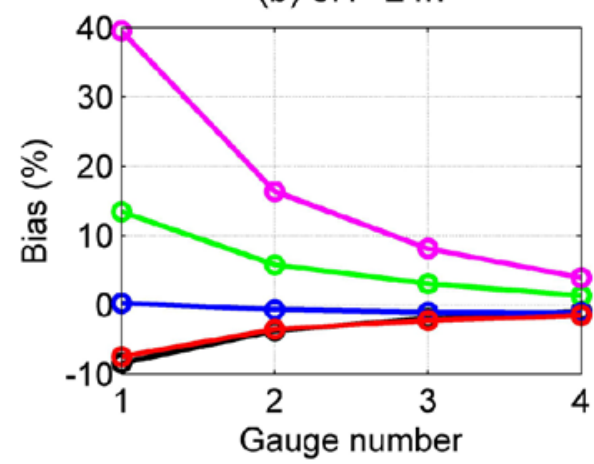

(d) $0.8^{\circ} 24 \mathrm{~h}$

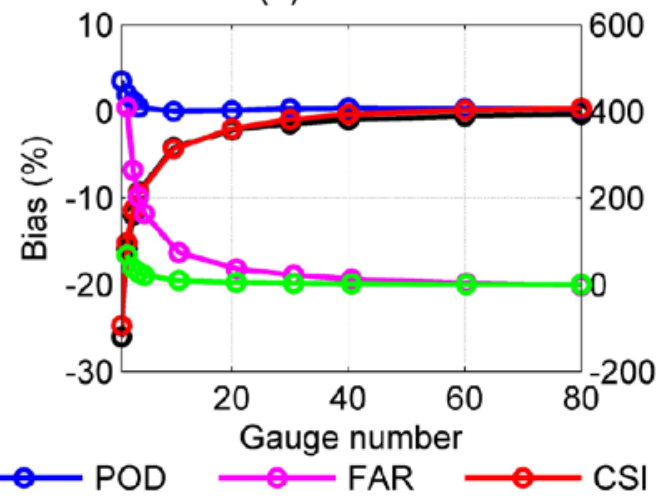

Figure 11. Errors of CC, NRMSE, POD, FAR and CSI with different numbers of gauges contained in

478 each grid pixel at $0.1^{\circ} / 0.8^{\circ}$ and $1 / 24$ h scales. The numbers increase from one to $80 \%$ of T2 in Table 1.

479 The second Y-axis on the right in (d) is for the biases of NRMSE and FAR.

$480 \quad 3.4$ Error function of spatiotemporal scales and gauge numbers

$481 \triangle$ MET of all metrics except BIAS can be estimated using Eq. (8). Inputs were acquired

482 with the spatial scale changing from $0.1^{\circ}$ to $0.8^{\circ}$, the temporal scale changing from $1 \mathrm{~h}$ to

$48324 \mathrm{~h}$, and the number of gauges in each grid pixel changing from one to $80 \%$ of $\mathrm{T} 2$.

484 Parameters were derived using the least square method (Table 2). Coefficients of

485 determination $\left(R^{2}\right)$ of CC, NRMSE, FAR and CSI are above 0.9, indicating that the error 
486 function fits well for the four metrics. However, POD exhibits much lower $R^{2}$, which is 487 as expected because of its irregular patterns in Section 3.2 and 3.3.

488 The sign of parameter $a$ represents whether the metric is underestimated or overestimated. 489 The signs of parameter $b, c$, and $d$ represent whether the magnitude of $\triangle$ MET is 490 positively or negatively correlated with $s, t$, and $N_{s t}$, respectively. $\Delta$ MET based on 491 sparse gauge networks can be estimated by combining Eq. (8) and parameters in Table 2 492 at arbitrarily spatiotemporal scales. For example, in the evaluation of the TMPA products 493 at the $0.25^{\circ}$ scale with each grid pixel only containing one gauge, $\Delta \mathrm{CC}$ could be around $494-26 \%$ and $-14 \%$ at $3 \mathrm{~h}$ and $24 \mathrm{~h}$ scales, respectively. At the IMERG-L resolution $\left(0.1^{\circ}\right.$ and $4950.5 \mathrm{~h}), \Delta \mathrm{CC}$ decreases from $-16 \%$ to $-5 \%$ as the gauge number in each pixel increases 496 from one to three. At the reanalysis resolution (e.g., $0.5^{\circ}$ and $6 \mathrm{~h}$ ), $\Delta \mathrm{CC}$ decreases from $497-46 \%$ to $-8 \%$ as the gauge number in each pixel increases from one to five.

498 Table 2 Parameters of the nonlinear error function used to estimate the errors of metrics and the 499 coefficients of determination $\left(R^{2}\right)$ for the fitting.

\begin{tabular}{cccccc}
\hline Parameters & CC & NRMSE & POD & FAR & CSI \\
$a$ & -161.45 & 256.95 & 52.82 & 521.90 & -99.59 \\
$b$ & 1.08 & 1.19 & 1.36 & 1.52 & 1.08 \\
$c$ & -0.29 & -0.16 & -0.48 & 0.20 & -0.15 \\
$d$ & -1.09 & -1.13 & -0.98 & -1.07 & -1.07
\end{tabular}




\section{$\begin{array}{llllll}R^{2} & 0.97 & 0.95 & 0.45 & 0.91 & 0.92\end{array}$}

500 Eq. (9) can be used to calculate the gauge density at different spatiotemporal scales to

501 achieve metrics within a predefined error level. Figure 12 shows the minimum number of

502 gauges in each grid pixel to control $\triangle \mathrm{MET}$ within $\pm 5 \%$. For CC, NRMSE, and CSI, the

503 number increases as the temporal scale decreases and the spatial scale increases. For

504 example, to control $\Delta \mathrm{CC}$ within $\pm 5 \%$, the minimum gauge number is 2.5 at the $0.1^{\circ}$ and

$5051 \mathrm{~h}$ scale corresponding to products such as IMERG-L and GSMaP. The number

506 increases to 4.6 at the $0.25^{\circ}$ and 3 h scale corresponding to products such as TMPA and

507 CMORPH. For FAR, the number increases as the temporal scale decreases and the spatial

508 scale increases (Figure 12c). It is noted that the minimum required gauge number of FAR

509 is much larger than that of other metrics.

(a) $\mathrm{CC}$

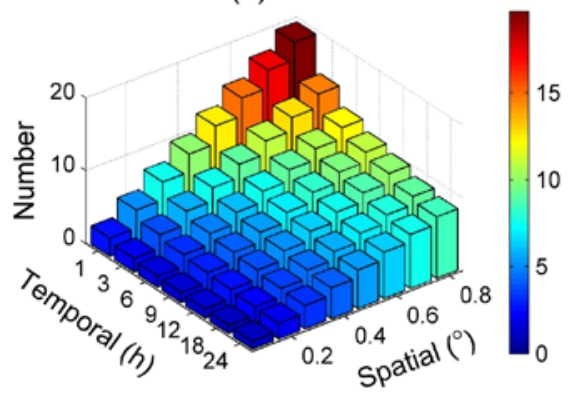

(c) FAR

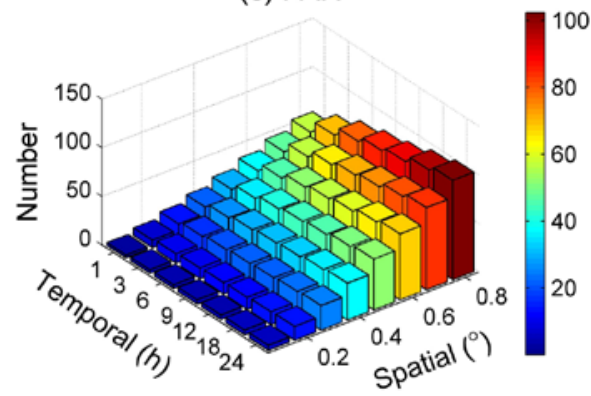

(b) NRMSE

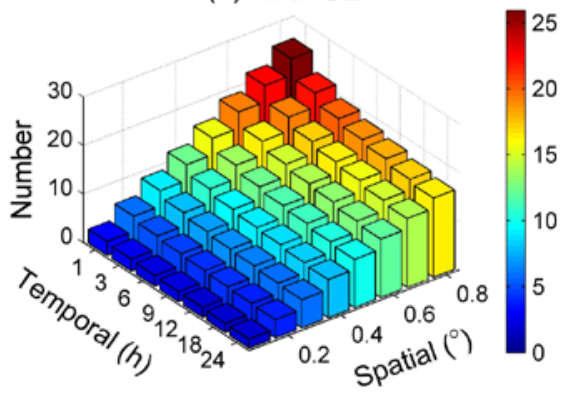

(d) CSI

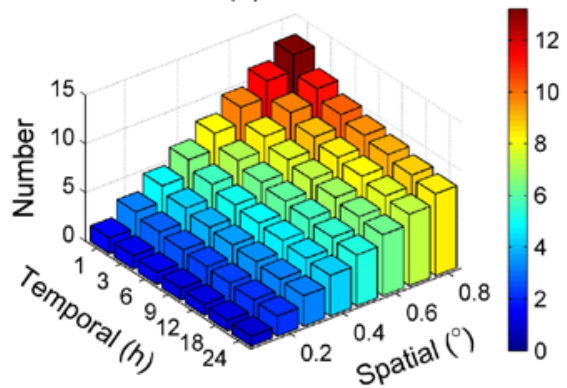


511 Figure 12. Minimum numbers of gauges in each grid pixel at different spatiotemporal scales to

512 control the biases of (a) CC, (b) NRMSE, (c) FAR, and (d) CSI within $\pm 5 \%$.

\section{4. Discussion}

\section{$514 \quad 4.1$ Applicability of the error function}

515 The error function fits quite well with CC, NRMSE, FAR, and CSI, worse for POD, and

516 is not suitable for BIAS. Fortunately, CSI is a more comprehensive contingency metric

517 combining the advantages of both POD and FAR. BIAS is a unique metric which suffers

518 little from sparse gauges. However, it should be noted that if the BIAS is relatively small

519 in the evaluation, the reliability of its sign (i.e., negative or positive) should be checked

520 by considering the locations and density of gauges. There are other metrics such as the

521 frequency of hit (FOH) and Heidke skill score (HSS), which are not included in this study.

522 We believe that most metrics used to indicate the performance of satellite precipitation

523 products tend to be worse based on sparse gauge networks. However, the applicability of

524 the error function (Eq. (8)) for other metrics still needs validation.

525 Although the study was conducted in the Ganjiang River basin, the error function could

526 also be applicable in other regions, particularly for those with similar topography and

527 climate. For regions where precipitation has a stronger variability in space and time at the

528 sub-grid scale, the errors of metrics based on sparse gauge networks would be larger, and

529 vice versa. Therefore, it is possible to add a parameter (such as coefficient of variation) in

530 the error function to represent regional variability and to make the function applicable in 
531 various regions. Furthermore, cold seasons are different from warm seasons concerning

532 precipitation characteristics, particularly in high latitude and high altitude regions where

533 snowfall dominates in winter. Gauge undercatch is particularly important for snowfall.

534 Meanwhile, satellite retrievals are more difficult over regions with snow cover and not

535 good enough at snowfall estimation. Finally, the current study period is relatively short.

536 The general conclusion should be consistent even for a longer period because the

537 limitation of gauge observations is inherent. However, more robust parameters of the

538 error function could be acquired if longer data series are employed. Further studies are

539 needed to explore the effect of gauge densities on the evaluation of rainfall/snowfall

540 using long series of gauge or radar observations.

541 For all remote sensing technologies and models providing areal estimates (e.g.,

542 evapotranspiration, soil moisture, and snow cover), their performance could also be

543 underestimated if the evaluation is conducted using point-scale measurements. The

544 degree of underestimation depends on the spatiotemporal scales and the number of

545 gauges in each grid pixel.

\section{$546 \quad 4.2$ Error variation and uneven gauge distributions}

547 The error function estimates the mean error of metrics based on combinations of random

548 sparse gauge networks in this study. As shown in Figure 6, overall CC values based on

549 sparse gauge networks follow the normal distribution. Other metrics are similar with CC

550 respecting the distribution. The error function approximates the mean or expectation of 
551 the normal distribution. However, in actual situations it is hard to know the actual

552 performance of a sparse gauge network. Errors of metrics calculated using the error

553 function can act as the reference but are not entirely the same as the actual numbers.

554 Therefore, the variance of metrics should be estimated in future studies to enable

555 estimation of bias range.

556 Another issue is that the number of gauges in each grid pixel is not the same for a specific

557 gauge network, which is different from the ideal situation in this study. However, the

558 uneven distribution of gauges is not beneficial to reducing the biases of gauges. Metrics

559 based on uneven gauge networks are generally worse than even gauge networks with the

560 same total gauges. We experimented by generating gauge networks with half grid pixels

561 containing one gauge and half grid pixels containing three gauges. $\triangle$ MET based on such

562 gauge networks is larger than that based on a gauge network with all grid pixels

563 containing two gauges. For example, the mean $\triangle$ NRMSE is $35 \%$ based on the uneven

564 gauge network, and $29 \%$ based on the even gauge network. The reason is that marginal

565 benefits become smaller as the gauge number increases. The adverse effect of pixels with

566 fewer gauges overwhelms the favorable effect of pixels containing more gauges.

\section{5. Conclusion}

568 Gauge networks with limited numbers of gauges in each grid pixel generally

569 underestimate the quality of satellite products because point-scale observations of scarce

570 gauges cannot perfectly represent areal precipitation. This study focuses on the errors of 
571 six metrics based on sparse networks which exhibit different spatiotemporal patterns.

572 Errors of CC, NRMSE, and CSI become larger as the spatial scale increases and temporal

573 scale decreases, while the error of FAR becomes larger as the temporal scale increases. In

574 contrast to other metrics, POD is overestimated by sparse gauge networks compared to

575 highly dense networks at 3-24 h scales because point-scale gauge observations cause

576 substantial uncertainties in calculating hit and miss event numbers. As a whole, among

577 the three contingency metrics, CSI is the most robust. Particularly, BIAS values based on

578 sparse gauge networks fluctuate around the value based on dense gauge network.

579 Therefore, it could be difficult to judge whether satellite products overestimate or

580 underestimate precipitation in a domain if BIAS values are not very large and gauges are

581 sparse.

582 We propose a nonlinear error function which expresses the spatiotemporal errors of CC,

583 NRMSE, FAR, and CSI pretty well with $R^{2}$ around or above 0.9 . The function can also be

584 used to estimate the required number of gauges in each grid pixel to deliver a predefined

585 error level. For example, to control the bias of CC within $\pm 5 \%$, the minimum gauge

586 number in each grid pixel is 2.5 at the $0.1^{\circ}$ and $1 \mathrm{~h}$ scale. The quantitative analysis in this

587 study is helpful to obtain more reliable information on the quality of satellite products.

588 The concept can also guide the design of optimized gauge networks for field studies. As

589 the results could depend on region and season, future studies should explore the

590 sensitivity of the results to variations in topography, climate conditions, and precipitation 
591 phase. The formula of the error function can potentially be improved to include more

592 metrics and to account for regional and seasonal variations.

593

\section{Acknowledgement:}

595 This study was financially supported by the National Key Research and Development 596 Program of China (2016YFE0102400), and the National Natural Science Foundation of 597 China (Grant No. 71461010701, 91437214 and 91547210). A. Behrangi is supported by 598 NASA Energy and Water Cycle Study (NNH13ZDA001N-NEWS) and NASA weather 599 (NNH13ZDA001N-Weather) programs. Part of the research was carried out at the Jet 600 Propulsion Laboratory, California Institute of Technology, under a contract with the

601 National Aeronautics and Space Administration. Additional support came from a 602 scholarship from the China Scholarship Council (CSC). Editors' and reviewers' 603 comments for improving this manuscript are highly appreciated.

604

\section{References:}

606 Adam, J.C., Lettenmaier, D.P., 2003. Adjustment of global gridded precipitation for 607 systematic bias. Journal of Geophysical Research: Atmospheres, 108(D9). $608 \quad$ DOI:10.1029/2002jd002499

609 Asong, Z.E., Razavi, S., Wheater, H.S., Wong, J.S., 2017. Evaluation of Integrated 
613 Behrangi, A., Gardner, A.S., Reager, J.T., Fisher, J.B., 2017. Using GRACE to constrain precipitation amount over cold mountainous basins. Geophysical Research Letters, 44(1): 219-227. DOI:10.1002/2016gl071832

616 Behrangi, A. et al., 2011. Hydrologic evaluation of satellite precipitation products over a mid-size basin. Journal of Hydrology, 397(3-4): 225-237. DOI:10.1016/j.jhydrol.2010.11.043

619 Ciabatta, L. et al., 2015. Integration of Satellite Soil Moisture and Rainfall Observations over the Italian Territory. Journal of Hydrometeorology, 16(3): 1341-1355. DOI:10.1175/jhm-d-14-0108.1

622 Cohen Liechti, T., Matos, J.P., Boillat, J.L., Schleiss, A.J., 2012. Comparison and evaluation of satellite derived precipitation products for hydrological modeling of the Zambezi River Basin. Hydrology and Earth System Sciences, 16(2): 489-500. DOI:10.5194/hess-16-489-2012

626 Gao, Y.C., Liu, M.F., 2013. Evaluation of high-resolution satellite precipitation products using rain gauge observations over the Tibetan Plateau. Hydrology and Earth System Sciences, 17(2): 837-849. DOI:10.5194/hess-17-837-2013

629 Hong, Y., Hsu, K.-l., Moradkhani, H., Sorooshian, S., 2006. Uncertainty quantification of 
propagation into hydrologic response. Water Resources Research, 42(8).

633 Hu, Q.F. et al., 2014. Multi-scale evaluation of six high-resolution satellite monthly

634 rainfall estimates over a humid region in China with dense rain gauges.

635 International Journal of Remote Sensing, 35(4): 1272-1294.

DOI:10.1080/01431161.2013.876118

637 Huffman, G.J. et al., 2017. NASA Global Precipitation Measurement (GPM) Integrated Multi-satellitE Retrievals for GPM (IMERG). Algorithm Theoretical Basis Document (ATBD), NASA/GSFC, Greenbelt, MD, USA (https://pmm.nasa.gov/sites/default/files/document_files/IMERG_ATBD_V5.1b.p df).

642 Huffman, G.J. et al., 2007. The TRMM Multisatellite Precipitation Analysis (TMPA):

643 Quasi-Global, Multiyear, Combined-Sensor Precipitation Estimates at Fine Scales.

644 Journal of Hydrometeorology, 8(1): 38-55. DOI:10.1175/jhm560.1

645 Jensen, N.E., Pedersen, L., 2005. Spatial variability of rainfall: Variations within a single

646 radar pixel. Atmospheric Research, $\quad$ 77(1-4): 269-277. DOI:10.1016/j.atmosres.2004.10.029

648 Joyce, R.J., Janowiak, J.E., Arkin, P.A., Xie, P.P., 2004. CMORPH: A method that produces global precipitation estimates from passive microwave and infrared data at high spatial and temporal resolution. Journal of Hydrometeorology, 5(3): 487-503. DOI:Doi 10.1175/1525-7541(2004)005<0487:Camtpg>2.0.Co;2 
652 Kubota, T. et al., 2007. Global Precipitation Map Using Satellite-Borne Microwave 653 Radiometers by the GSMaP Project: Production and Validation. IEEE 654 Transactions on Geoscience and Remote Sensing, 45(7): 2259-2275. $655 \quad$ DOI:10.1109/tgrs.2007.895337

656 Li, Z., Yang, D., Hong, Y., 2013. Multi-scale evaluation of high-resolution multi-sensor 657 blended global precipitation products over the Yangtze River. Journal of 658 Hydrology, 500: 157-169. DOI:10.1016/j.jhydrol.2013.07.023

659 Ma, Y. et al., 2016. Similarity and Error Intercomparison of the GPM and Its 660 Predecessor-TRMM Multisatellite Precipitation Analysis Using the Best Available 661 Hourly Gauge Network over the Tibetan Plateau. Remote Sensing, 8(7): 569. $662 \quad$ DOI:10.3390/rs8070569

663 Ma, Y., Zhang, Y., Yang, D., Farhan, S.B., 2015. Precipitation bias variability versus 664 various gauges under different climatic conditions over the Third Pole 665 Environment (TPE) region. International Journal of Climatology, 35(7): 666 1201-1211. DOI:10.1002/joc.4045

667 Mandapaka, P.V., Krajewski, W.F., Ciach, G.J., Villarini, G., Smith, J.A., 2009. 668 Estimation of Radar-Rainfall Error Spatial Correlation. Advances in Water 669 Resources, 32(7): 1020-1030. DOI:10.1016/j.advwatres.2008.08.014]

670 Mandapaka, P.V., Villarini, G., Seo, B.-C., Krajewski, W.F., 2010. Effect of radar-rainfall 671 uncertainties on the spatial characterization of rainfall events. Journal of 672 Geophysical Research, 115(D17). DOI:10.1029/2009jd013366 
673 Peleg, N., Ben-Asher, M., Morin, E., 2013. Radar subpixel-scale rainfall variability and 674 uncertainty: lessons learned from observations of a dense rain-gauge network. 675 Hydrology and Earth System Sciences, 17(6): 2195-2208. 676 DOI:10.5194/hess-17-2195-2013

677 Rudolf, B., Hauschild, H., Rueth, W., Schneider, U., 1994. Terrestrial precipitation 678 analysis: Operational method and required density of point measurements, Global 679 precipitations and climate change. Springer, pp. 173-186.

680 Salio, P., Hobouchian, M.P., García Skabar, Y., Vila, D., 2015. Evaluation of 681 high-resolution satellite precipitation estimates over southern South America 682 using a dense rain gauge network. Atmospheric Research, 163: 146-161. 683 DOI:10.1016/j.atmosres.2014.11.017

684 Shen, Y., Xiong, A., Wang, Y., Xie, P., 2010. Performance of high-resolution satellite 685 precipitation products over China. Journal of Geophysical Research, 115(D2). 686 DOI:10.1029/2009jd012097

687 Shen, Y., Zhao, P., Pan, Y., Yu, J.J., 2014. A high spatiotemporal gauge-satellite merged 688 precipitation analysis over China. J Geophys Res-Atmos, 119(6): 3063-3075. 689 DOI:10.1002/2013jd020686

690 Tan, J., Petersen, W.A., Tokay, A., 2016. A Novel Approach to Identify Sources of Errors 691 in IMERG for GPM Ground Validation. Journal of Hydrometeorology, 17(9): 692 2477-2491. DOI:10.1175/Jhm-D-16-0079.1

693 Tan, M.L., Duan, Z., 2017. Assessment of GPM and TRMM Precipitation Products over 
696 Tang, G., Long, D., Hong, Y., 2016a. Systematic Anomalies Over Inland Water Bodies of High Mountain Asia in TRMM Precipitation Estimates: No Longer a Problem for the GPM Era? IEEE Geoscience and Remote Sensing Letters, 13(12): 1762-1766. DOI:10.1109/lgrs.2016.2606769

700 Tang, G., Ma, Y., Long, D., Zhong, L., Hong, Y., 2016b. Evaluation of GPM Day-1 IMERG and TMPA Version-7 legacy products over Mainland China at multiple spatiotemporal scales. Journal of Hydrology, 533: 152-167. DOI:10.1016/j.jhydrol.2015.12.008

704 Tang, G. et al., 2016c. Statistical and Hydrological Comparisons between TRMM and GPM Level-3 Products over a Midlatitude Basin: Is Day-1 IMERG a Good Successor for TMPA 3B42V7? Journal of Hydrometeorology, 17(1): 121-137. DOI:10.1175/jhm-d-15-0059.1

708 Tian, Y., Peters-Lidard, C.D., 2010. A global map of uncertainties in satellite-based precipitation measurements. Geophysical Research Letters, 37(24): n/a-n/a. DOI:10.1029/2010gl046008

711 Vicente-Serrano, S.M., Saz-Sanchez, M.A., Cuadrat, J.M., 2003. Comparative analysis of 712 interpolation methods in the middle Ebro Valley (Spain): application to annual 713 precipitation and temperature. Climate Res, 24(2): 161-180. DOI:DOI 
715 Villarini, G., Mandapaka, P.V., Krajewski, W.F., Moore, R.J., 2008. Rainfall and sampling

716 uncertainties: A rain gauge perspective. Journal of Geophysical Research, 717 113(D11). DOI:10.1029/2007jd009214

718 Wood, S.J., Jones, D.A., Moore, R.J., 2000. Accuracy of rainfall measurement for scales

719 of hydrological interest. Hydrology and Earth System Sciences, 4(4): 531-543.

720 Xie, P.P., Arkin, P.A., 1997. Global precipitation: A 17-year monthly analysis based on

721 gauge observations, satellite estimates, and numerical model outputs. Bulletin of

722 the American Meteorological Society, 78(11): 2539-2558. DOI:Doi

$723 \quad$ 10.1175/1520-0477(1997)078<2539:Gpayma>2.0.Co;2

$724 \mathrm{Xu}$, R. et al., 2017. Ground validation of GPM IMERG and TRMM 3B42V7 rainfall

725 products over southern Tibetan Plateau based on a high-density rain gauge

726 network. Journal of Geophysical Research: Atmospheres, 122(2): 910-924.

727 DOI:10.1002/2016jd025418

728 Xue, X. et al., 2013. Statistical and hydrological evaluation of TRMM-based

729 Multi-satellite Precipitation Analysis over the Wangchu Basin of Bhutan: Are the

$730 \quad$ latest satellite precipitation products 3B42V7 ready for use in ungauged basins?

731 Journal of Hydrology, 499: 91-99. DOI:10.1016/j.jhydrol.2013.06.042

732 Yang, D.Q., Ohata, T., 2001. A bias-corrected Siberian regional precipitation climatology.

733 Journal of Hydrometeorology, 2(2): 122-139. DOI:Doi

734 10.1175/1525-7541(2001)002<0122:Abcsrp>2.0.Co;2

735 Yong, B. et al., 2014. Intercomparison of the Version-6 and Version-7 TMPA precipitation 
products over high and low latitudes basins with independent gauge networks: Is the newer version better in both real-time and post-real-time analysis for water resources and hydrologic extremes? Journal of Hydrology, 508: 77-87. DOI:10.1016/j.jhydrol.2013.10.050

740 Yong, B. et al., 2010. Hydrologic evaluation of Multisatellite Precipitation Analysis

741 standard precipitation products in basins beyond its inclined latitude band: A case

742 study in Laohahe basin, China. Water Resources Research, 46(7).

743 DOI:10.1029/2009wr008965

744 Yong, B. et al., 2013. First evaluation of the climatological calibration algorithm in the 745 real-time TMPA precipitation estimates over two basins at high and low latitudes.

746 Water Resources Research, 49(5): 2461-2472. DOI:10.1002/wrcr.20246 